\title{
Silent Ships - a New Challenge for the Shipbuilding Industry
}

\author{
Stefan Weyna \\ Marine Technology and Transport Faculty, West-Pomeranian University of Technology, Szczecin, Poland \\ Email address: \\ Stefan.Weyna@zut.edu.pl \\ To cite this article: \\ Stefan Weyna. Silent Ships - a New Challenge for the Shipbuilding Industry. American Journal of Modern Physics. \\ Vol. 10, No. 4, 2021, pp. 71-92. doi: 10.11648/j.ajmp.20211004.12
}

Received: July 5, 2021; Accepted: July 28, 2021; Published: August 12, 2021

\begin{abstract}
This publication presents new research techniques for noise abatement on ships obtaining vibroacoustic parameters of passenger and crew accommodations for newly designed ships. The construction of low-noise ships is a new technical challenge for naval architects, shipbuilders and shipowners. It also requires a new way of looking at these problems. The suggested new research methods based on sound intensity (SI) and laser anemometry techniques (PIV, LDA) in combination with graphical presentation as acoustical imaging (AI) of the field distribution of acoustic wave flows, gives a full range of interpretation possibilities of energy effects and the precautions taken. This will ensure vibro-acoustic optimisation of the product and meet design and standardisation objectives for noise and vibration levels on board. SI analyses are supported by acoustic orthogonal decomposition (AOD) of disturbed acoustic vector fields with a turbulent structure. The innovative SIAOD method, combined with acoustical imaging (AI) of the results, allows a comprehensive interpretation of noise causes and becomes an highly effective tool for noise abatement on board ships. With a new, holistic approach to explain the mechanism of penetration of structure-borne and airborne sounds into ship interiors, it creates a balance of acoustic power of local sources of noise, which is the basis for the choice of methods for noise reduction. The author describes the mechanism of noise generation inside shipboard accommodations and in shipboard HVAC systems.
\end{abstract}

Keywords: Vibroacoustics, Ship Noise, Sound Intensity, Acoustical Imaging

\section{Introduction}

There are certain acoustic phenomena that significantly affect our quality of life. The negative side of acoustics is noise. Noise is the most commonly cited form of environmental pollution. In recent decades, the effects of noise on people have been studied intensively. Much of the experimental researches are focused on finding better ways to identify noise sources and noise transport paths. Investigators continue to work on improvement processing techniques that can be used to visualize noise sources to better understand complicated machinery and other noise generators. Then models are built to observe the dynamics of the spread of noise-generating acoustic phenomena. Finally, methods of noise reduction are developed. Other experimental research is focused on developing methods, using sound and vibration signatures, for diagnosing and predicting potential problems. These methods utilize advanced signal processing and data reduction techniques to identify fault-sensitive parameters.
The noise generation of turbulent flows over surfaces, in ducts and around edges of bluff bodies of structures is a general theoretical and design problem with important implications in protecting humanity from excessive noise. Turbulent boundary layers near the trailing-edge of a surface are known to generate intense surface pressure fluctuations as well as broadband scattering high-profile noise level.

Researchers try to understand and quantify human reaction to noise, to identify the characteristics of sounds that people respond to and how these characteristics combine to give a person an overall impression of sound. These psychoacoustic models are combined with acoustical source-path models to have people-focused optimization of acoustic environments, e.g. the sound propagation outdoors in a complex urban and suburban environment. Recent applications include quantifying annoyance due to environmental noise, the sound quality of refrigerators, HVAC systems, diesel engines and chillers, and the impact of vehicle interior noise on speech intelligibility in older drivers. Laboratory research is strongly 
interested in system performance in practical environments, and hence techniques are being developed for in-situ determination of acoustical properties of sound absorption materials. This includes the design and optimization of acoustical characteristics of acoustical materials and composite systems of such materials. An important outcome of much of the acoustics group's research is the development of new noise control treatment methods. Applications include fuselage-trim assemblies for aircraft, car door treatments and low-noise wall construction for buildings.

The acoustic problem relating to shipbuilding products has grown significantly along with social impact on the protection of the marine environment [1], followed by changes in the requirements of classification societies for the construction of silent ships. For naval architects, ship builders and owners the problem of acoustic protection on ships and offshore platforms has become a new technical and economical challenge.

\section{Quiet Ships in the Shipbuilding Market}

\subsection{New Recommendations and Regulations in Shipbuilding}

Research on noise abatement on board has a long history. The problem of effective noise reduction on ships remains important and stimulates cooperation between designers, shipbuilders and shipowners in order to work out compromise solutions that meet the requirements of national vessel registration authorities. Today research in this field is focused on environmental protection and reduction of ship vibration and noise levels. Another goal is to create more friendly working environment, protect the health crews, enhance passenger comfort at sea and protect the marine environment.

Observing the domestic and foreign shipbuilding industry, one can notice a breakthrough change in the assortment of ships delivered. Changes in the profile of ship design and production result from increased interest of ship owners in the construction of luxury ships. Moreover, legislative changes introduced recently by international maritime organisations increase the protection of seafarers' work and introduce new regulations for the protection of the marine environment. All these changes have also led to the introduction of a new classification of ships for this group Quiet Ships. Ship classification is defined as the process of verifying ship standards against a set of requirements made mandatory by international regulations.

New ships intended for international trade must satisfy international safety and environmental regulations established by the International Maritime Organisation (IMO) and International Labour Organisation (ILO). The regulations also contribute to the prevention of exposure to hazardous noise levels on board ships. These include international conventions such as Safety of Life at Sea. (SOLAS, [2]) and Prevention of Pollution from Ships
(MARPOL). In 2012 IMO adopted a regulation in the International Convention for the Safety of Life at Sea (SOLAS) requiring new builds to reduce on-board noise and to protect personnel from noise, in accordance with the Code on Noise Levels on Board Ships. The Code sets out mandatory maximum noise level limits for machinery spaces, control rooms, workshops, accommodation and other spaces.

We are just starting to make sure that new ships are environmentally friendly. Studies have shown that underwater-radiated noise from commercial ships may have both short and long-term negative consequences on marine life, especially marine mammals. The issue of underwater noise and impact on marine mammals was first raised at IMO in 2004. It was noted that continuous anthropogenic noise in the ocean was primarily generated by shipping. Since ships routinely cross international boundaries, management of such noise required a coordinated international response. In 2008, the IMO Marine Environment Protection Committee (MEPC) agreed to develop non-mandatory technical guidelines to minimize the introduction of incidental noise from commercial shipping operations into the marine environment to reduce underwater noise and impact on marine mammals' life.

Closer monitoring of seafarers' health and safety is required as is an enhanced international regulatory regime to narrow the wide gap between best and worst industry practices. Innovative research techniques proposed by this author can help ship designers and engineers make the best decisions in terms of selection of the optimal marine equipment design in terms of vibroacoustics. The technical development of ships has successively given rise to increased problems regarding the vibroacoustic environment on board. For the builders of new merchant and passenger ships, meeting the standards for vibroacoustic protection, especially for ships of the Quiet Ships class, is a major design challenge. Ships of this class are expected not only to be crew-friendly and comfortable for passengers, but also to reduce the degree of acoustic hazards to which marine life organisms of living seas and oceans are exposed. New research techniques proposed herein include the development of research methods allowing ship designers to devise effective technical solutions to reduce noise levels on board to the levels required by the classification codes.

\subsection{Legislation and the Marine Directive}

The European Union is seeking the development by the IMO and standardization bodies of relevant international standards, including detailed technical specifications and testing standards for ship equipment, the use of which is a fundamental condition for enhancing maritime safety and preventing marine pollution. The aim of the European Union's ambitious Marine Strategy Framework Directive is to protect more effectively the marine environment across Europe. The Commission also produced a set of detailed criteria and methodological standards to help Member States implement the Marine Directive - "The European Marine Strategy Framework Directive" (MSFD) requires European 
states to maintain their marine waters in "Good Environmental Status" (GES) [3]. The Directive enshrines in a legislative framework the ecosystem approach to the management of human activities having an impact on the marine environment, integrating the concepts of environmental protection and sustainable use. This is in line with the European Commission and IMO. One of the main drivers of the record is to help European stakeholders to meet the requirements of the Marine Strategy Framework Directive. This aims to improve the environmental status of European waters by proposing mitigation solutions, and their effectiveness was to be confirmed by 2020. The Marine Directive was adopted on 2008 and amended in 2017.

\subsection{The New Classification of Silent Ships}

Ship classification provides a point of reference for ship safety and reliability. It is represented by a class, entered on classification certificates and transcribed in the register of ships published periodically by a classification society. Quiet Ships classification notations shall be assigned to ships to determine the relative rules of classification societies for the assignment and maintenance of class. The classification defines restrictions on vibration and noise levels of marine machinery and equipment and sets the limit levels for underwater noise radiation.

Meeting the classification requirements is a challenge for ship designers and builders to increase the market value of the ship in terms of data quality, improving working conditions for ship crews, increase passenger comfort and improve the protection of the marine environment. This confirms that shipyards and ship owners are credible partners genuinely committed to reducing the harmful effects of shipbuilding on the environment.

The largest European classification societies, such as BV (2014), DNV-GL (2015), LR (2007) and RINA (2014) have recently launched their own new regulations on silent shipbuilding [4-7]. For instance, Bureau Veritas has launched an underwater noise reduction notation NR614 Underwater Radiated Noise (URN) a voluntary notation intended to assist shipbuilders and operators reduce underwater noise radiating from ships. The comprehensive set of standards and measuring services aims to control and limit the environmental impact on marine fauna of all self-propelled ships. Although the notation NR614 stands for underwater noise protection, these requirements can only be met, if the interior of the ship is quiet enough. Reducing underwater noise directly contributes to reduced noise and vibration levels on board, which improves passenger and crew comfort. Therefore, the new BV COMFORT Class includes additional requirements in the regulations labelled as $\mathrm{COMF}+$, applicable to five different hazard areas categories: COMF+ Insulation index, Impact index, Emergence, Intermittent noise and Intelligibility. $\mathrm{COMF}+$ standards set out noise limits criteria for different on board spaces for the expected normal operating conditions of the vessel.

Today, the COMFORT class, supported by Bureau Veritas' qualified noise and vibration teams, is the most up-to-date and complete set of verifiable standards to ensure a comfortable environment on board any type of merchant ship: passenger ships (such as cruise ships, ferries or ro-pax), cargo ships, fishing vessels, tugs, yachts and mega yachts. The living and working spaces of a ship shall be protected from excessive noise.

Partitions in the living quarters of the ship, floors and doors shall comply with the requirements of COMFORT class. In the process of identification and selection of class variants, many factors that contribute to the reduction of vibrations and noise on board ships are taken into account. These include efficiency, costs, feasibility of implementation, availability, etc.

Another example of classification standards introduced for silent vessels are the DNV GL regulations. For the protection of ships from vibrations and noise, DNV GL proposals to classify silent ships are found in "Quiet Ships - rules for ships classification" [4]. It offers on a voluntary basis the introduction of three voluntary class notations: Comfort Class, Vibration Class and Silent Class.

Vessels, which fulfill the requirements for Comfort Class offer objectively evaluated comfort of work and travel on a specific ship. A high level of comfort increases the performance and vigilance of the crew - consequently safer operation of ships. Acoustic hazard is a significant parameter for the ship's rating and reputation in the market. Comfort Class ships shall be numbered; from 1 to 3 . A rating from 1 to 3 reflects "high" to "acceptable" comfort standards. Ships are assigned a class notation COMF-V for noise and vibration and COMF-C for indoor climate. Additionally, rating number 3 ensures compliance with MSC 337 (91) on noise and ISO 6954 on vibration. Compliance to the rules is to be verified through measurements when the vessel is completed. Although the comfort class is a voluntary record of comfort assessment on board of different types of vessels, there is a strong emphasis on compliance with the record for this purpose by ship owners ordering cruise passenger ships, ropax and ferries.

Vibration Class includes vibration limits for various marine machines and equipment. It is based on the values obtained by numerous measurements. Ships meeting the requirements of the Vibration Class will be assigned VIBR class. Vibration class includes a comprehensive set of vibration criteria for the design, machinery and equipment installed on board ships. Ships designed to increase comfort by improving the acoustic climate in the home are $C$ and $V$ crn (crn-comfort rating number) certified.

The Silent Class provides owners of acoustically sensitive vessels with concise, realistic criteria for underwater noise emissions. It gives ship owners the opportunity to demonstrate an environmentally friendly ship with low noise levels emission to the marine environment. Ships meeting the requirements of the Silent Ship class are awarded the SILENT mark. Voluntary compliance with the provisions of this class guarantees a realistic low emission of underwater noise, which will affect the protection of marine fauna and flora [8]. A significant gap in our knowledge is our lack of 
understanding of the potential long-term and population-level impacts and the corresponding biological significance. Marine mammals use sound for navigation, food finding, communication and are particularly affected by the rising background noise levels from the growing global fleet of commercial and recreational shipping.

In the summary of the subject matter concerning the introduction of the new classification of ships, it should be noted that compliance with the class conditions places the ship's vibration and sound protection at the forefront of the design requirements. Although the protection of ships from excessive vibration and noise is not a new issue for designers, in this case it seems necessary to establish cooperation with specialists on these problems. Although for the time being the new classifications are not being used obligatorily, it seems that they will soon become mandatory in shipbuilding and become an indicator of the modernized product. The intensive work of European and global classification societies on the regulation of the construction of Quiet Ships shows that the problem is global and we should expect IMO's legislative provisions on the matter.

New requirements for marine acoustics stimulate theoretical and experimental research aimed at finding practical solutions for the shipbuilding industry. This is what this author suggested presenting his innovative research results, being confident that scientists can help designers, yards and operators to be ahead of the regulations.

\subsection{Management of the Working Environment in Ship Design}

The systematic management of the working environment of seafarers requires the development of the ship design process, including actions to control and verify that the design requirements recommended by IMO and ISO, are implemented. For modification projects, new management analyses or updating of existing analyses that are considered to be affected by the modification, shall be performed. In concept optimisation and design development, preventive safer design principles should always take priority over risk acceptance criteria for occupational safety, human error and health risks for crew and travel comfort for passengers. The risk analysis method shall be adapted to the applicable acceptance criteria.

Standardisation analyses are carried out to identify and assess occupational safety and health risks and potential problem areas related to various factors as input to design. When establishing noise level limits above $85 \mathrm{~dB}(\mathrm{~A})$, the individual worker's exposure time in noisy areas shall be considered in relation to noise exposure limits. The maximum possible noise level $110 \mathrm{~dB}(\mathrm{~A})$ should only be permitted for short-term inspections or work tasks that must be carried out as maintenance and service work. In such work, it must be possible to isolate noisy equipment during maintenance or other work in the area, and the repair team must be supplied with effective personal protective equipment against excessive vibration and noise. Details of the limit values for noise levels in working spaces and crew or passenger cabins are contained in the relevant IMO and ISO recommendations.

While the management of the working environment on ships has always been an element in the reasonable design of new vessels, designers of silent vessels must bear in mind that the priority for new vessels is to ensure even lower vibration and noise levels than those previously accepted. The existing professional practice of the designer must be enriched with new knowledge of modern techniques for combating industrial vibroacoustic hazards.

\section{Vortex Sound Theory and the Basics of Vector Acoustics}

\subsection{Seeking New Knowledge for Commercial Applications}

The search for new more effective techniques to eliminate environmental noise is a challenge not only for industry, but also stimulates the scientists to look for new knowledge that could bring some engineering innovations for these applications.

Nowadays acoustics is slightly different from traditional acoustics and research focuses on acoustic wave flow. Even though acoustics has always been seen as a field of interdisciplinary knowledge, the new approach highlights the strength of the relationship between acoustics and mechanics, fluid mechanics in particular. New acoustic theories are based on analyses of wave motion of physical particles in viscous media and therefore less importance is given to traditional analysis of acoustic pressure. All attention is focused on analyses of velocity of acoustic particles and energy impact that the flow is caused by dynamics of this motion in real media. The problem of acoustic flows is closely associated with physical phenomena of hydrodynamics and aeroacoustics. The behaviours of the acoustic particle in the field of the acoustic wave are described in analytical and numerical models with basic laws of momentum, mass and energy exchange, but adapted to the characteristics of the acoustic wave. Theoretical description of acoustic phenomena utilizes an extensive knowledge of fluid mechanics, and experimental research uses measurement tools known from the research on flows in liquids and gases (for example; PIV/LDV laser techniques and decomposition tools POD/DMD).

The science of sound related to the description of mechanical vibrations of an elastic medium is a field of knowledge about wave motion in gases, liquids and solids. The possibility of propagation of an acoustic wave is a characteristic property of all resilient media, but only some properties of liquids and gases allow defining the basic laws of propagation of an acoustic wave in the same mathematical forms. Liquids and gases are approximately treated as liquids in which the acoustic wave propagates without significant energy loss. This approach is commonly used in the continuous mechanics including problems of hydro- and thermodynamics and aeroacoustics. Although it seems that the propagation of sound waves in real media described in Lord Rayleigh's Theory of Sound and Theoretical Acoustics 
(1968) P. M. Morse and K. U. Ingard is a complete description of the sound theory, after several decades of stagnation we can observe the formation of a new flow theory of sound, a breakthrough in acoustics.

\subsection{The Vortex Sound Theory}

Any fluid that possesses intrinsic kinetic energy must possesses vorticity. For a vast number of flows, vorticity may be regarded as the ultimate source of the sound generated by the flow. However, it is widely recognized that any mechanism that produces sound can actually be formulated as a problem of intense turbulence velocity fluctuations. Turbulence phenomena are deeply non-linear in nature so they are difficult to theoretically describe and model.

The work of M. J. Lighthill in 1952 [9] was a breakthrough as it brought new knowledge of soundgenerating aerodynamic flows. In what today is called Lighthill analogy, describing the conversion of aeroacoustic energy into sound energy, the author uses Navier-Stocks equations to describe the mechanism of sound generation in the viscous medium (Lighthill's tensor), bringing the description of the sound source closer to real conditions. However, it was not a perfect model and other authors, Curle (1955), Powell (1965), Goldstein (1976), Möhring 1978, Ffowcs-Williams and Hawking (1969), Howe (2003) [1015], developed this model and became precursors of a new sound theory - Vortex Sound Theory (VST). By developing
Laighthill's mechano-acoustic analogue model and using numerical computational platforms CFD/FSI/CAA, the creators of VST theories have conveyed the old notion of acoustic flows associated with acoustics pressure measurements into a new, dynamically developing theory of acoustic wave motion described in a vectoral way, closely related to the theory of fluid mechanics. This changes the paradigm of classical acoustics - we move from pressure acoustics to vector acoustics [16-18]. Vector analyses give the possibility of imaging real acoustic wave flows and watching structural effects caused by the wave reaction on obstacles (Figure 1). Visualization of dispersion, reflection and scattering effects for various cases of eddy fields in real flows is the basis for analysis of noise sources and finding effective methods of noise reduction.

Today we can investigate the flow of acoustic waves in the air, similarly to aerodynamic or hydrodynamic flows. We must always emphasize that the physical cause of sound effects is the movement of particles, while streams of sound intensity are the energy determinant of this movement. For example, studies conducted using VSTs indicate that sound generated by the pairing of two inviscid finite-core-size twodimensional vortices is that the resulting sound field is a lateral quadrupole type and its axis rotates with non-uniform speed about the origin of the source region. Results also show that the accelerating and decelerating motions of the vortices are very important in the sound generation [19].

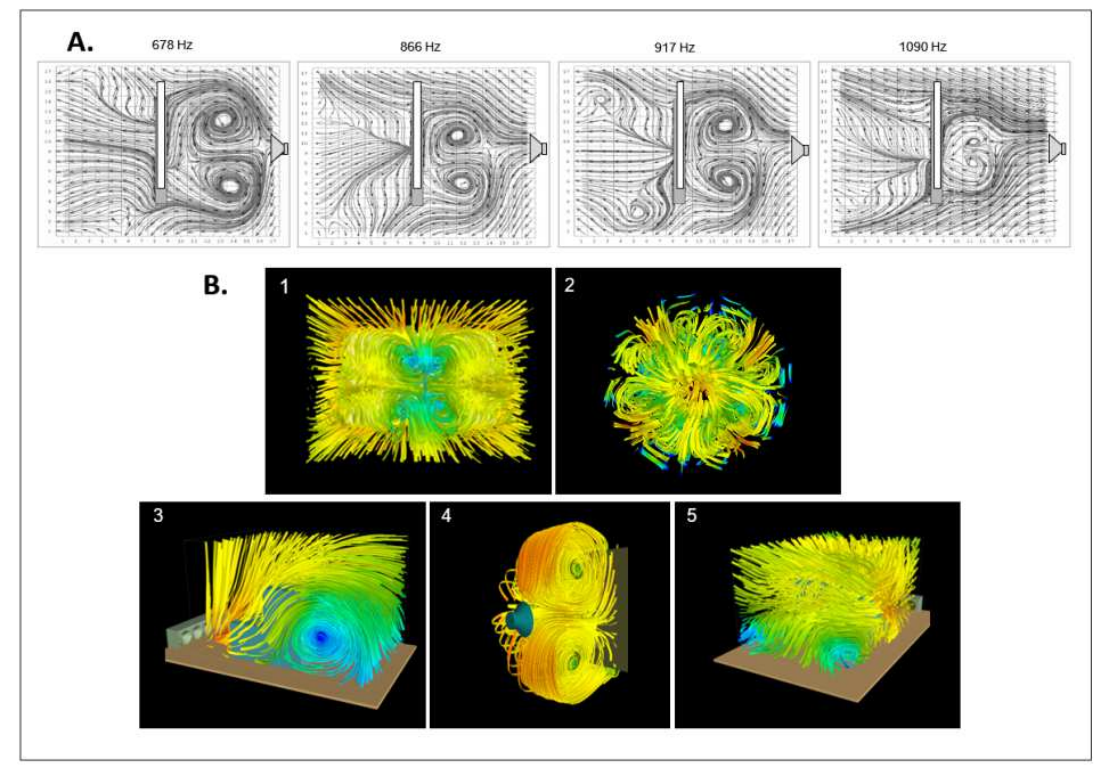

Figure 1. A. Intensity vectors maps and streamlines around a rectangular plate, $B$. Three-dimensional distribution of vortex sound intensity streamlines in a real acoustic flow fields: 1 - streamlines in a space behind a hard rectangular plate, 2 - image of $3 D$ streams at the outlet of a circular waveguide, 3 - vortex reactions of streamlines in the space behind the staircase, 4 - rotational effects of a wave reflected from a hard plate, 5 - Karman's streets behind a threedimensional wedge lying on a flat surface.

The source of noise is the disturbed character of the flow generated on geometrically varied structures introduced into the field of acoustic wave flow. Aerodynamic effects caused at the edges of structures and discontinuities of flow generating non-linear vortex effects and turbulence are associated with the birth of sound. The VST theory treats sound as a result of the interaction of independent eddies concentrated in disturbed space. The theory of acoustic vortex flows explains the mechanism of sound generation in the flow field. It shows that dynamic disturbances of the medium in the form of vortexes, rotation and turbulence, converting hydrodynamic energy into acoustic energy, 
become a sound source. The energetic treatment of the phenomenon of sound birth, as well as the definition of sound accepted by acoustics (sound is defined as mechanical vibrations in a resilient environment) emphasizes how important and close the relationship between acoustic knowledge and mechanics is. Mechanics confirming the consistency of fluid mechanics with acoustics, has produced new acoustic theories. Extended acoustic knowledge related to the use of previously developed knowledge in fluid mechanics is of great importance for acoustics in explaining the mechanism of sound generation and helps finding new optimal solutions to protect the environment from noise.

However, the modern VST theory of aerodynamic sound pioneered by Lighthill still wanted to understand the mechanisms of noise generated by unsteady motions of a fluid. The VST theory continues to be developed [20,21] and has industrial applications, also in ship acoustics (Quiet Ships). In our experimental studies we try to use the theoretical knowledge and the state-of-the-art solutions.

In the analysis of vector images of recorded waveforms in real acoustic flows, scientists focus on the analysis of areas with visible whirlpools, which, according to VST, signal the positioning of local noise sources. The effectiveness of technical noise reducing will be confirmed by the elimination, or at least a significant reduction the previously recorded vortex flow.

Acoustical imaging performed with the proposed SI technique should greatly help experts to draw correct conclusions in their future studies on vortex-free acoustic motion. Therefore, one of the objectives of our research $s$ to use the existing VST sound theory for the analysis of the rotationally disturbed real acoustic flows recorded and graphically visualized by the SI vector method. This acoustical imaging (AI) will allow us to understand the mechanism of sound formation and to quantitatively estimate recorded vortex phenomena. This knowledge will be useful in the choice of techniques to reduce noise from industrial sources.

\section{Sound Intensity as the Energy Parameter of the Acoustic Wave}

\subsection{The Sound and Noise}

To describe the acoustic perception of our environment, let us first clarify the notions of sound and noise. Sound is the sensation produced by very small movements of the medium's particles, which secondarily generate oscillating pressure changes in the surrounding air, and create an acoustic field. If the harmonic vibration of the medium reaches significant amplitudes, sound becomes noise. Sound is a wider concept than noise. Not all sounds are noise, but every noise is a sound! The development of modern knowledge of acoustics concerns all the dynamic phenomena that are considered in the physics of sounds, from infrasounds, through sounds heard by humans, to ultra- and hyper sounds.
Noise is defined as sound that is a subjective unwanted acoustic phenomenon whose level should not be exceeded for the sake of physical and mental health. For the whole human population certain maximum noise levels should not be exceeded. Considerations on ship noise begin with an outline of noise control strategies and an overview of the fundamental principles of acoustics, followed by an explanation of how noise is quantified, to provide a basis for understanding the applications discussed further in the article.

One of the important effects of aerodynamic flows is sound, which, by reaching high amplitudes, changes into annoying noise. The source of the noise is the disturbed nature of the flow arising from sudden discontinuities in limited flows (e.g. in rooms, in piping systems), or from objects appearing in the free field flow Aerodynamic flow effects induced at the edges of structures and at a discontinuity generate non-linear vortex movements and turn laminar flow into turbulent flow [22]. These phenomena involve energy, therefore energy parameters should be used in the measurement and analysis of acoustic flows. Sound intensity is one of such energy wave parameters.

\subsection{The Sound Intensity}

Sound waves propagating through a fluid result in energy transmission. As the process of sound propagation is cyclic, so is the power transmission and consequently an instantaneous and a time-average intensity may be defined. The possibility of direct measurement of sound intensity appeared in the early 1980s, when Frank Fahy [23] built a probe to measure sound intensity, a measuring transducer fulfilling the required metrological standards.

Sound intensity (SI) is a vector quantity determined as the product of sound pressure and the component of particle velocity in the direction of the intensity vector quantity $(I=$ $\left.u \cdot p,\left[\mathrm{~W} / \mathrm{m}^{2}\right]\right)$ as it is associated with the direction in which the energy is being transmitted. The instantaneous sound intensity, $I_{i}(r, t)$, in an acoustic field at a location given by the field vector, $r$ is a vector quantity describing the instantaneous acoustic power transmission per unit area in the direction of the vector particle velocity, $u(r, t)$. Having the possibility to directly measure the sound intensity flux in the acoustic field, we can finally directly determine the sound power radiated by the vibrating surfaces $\mathrm{S}$. The acoustic power $N_{a c}$ radiated by any acoustic source with a surface area of $\mathrm{S}$ is equal to $I \cdot S$. Another important parameter for vector acoustics is acoustic impedance $z_{a c}\left(z_{a c}=p / u=\rho c=I / u^{2}\right)$.

The new VST theory of acoustic vortex flows, introducing revolutionary changes to the principles and laws of classical acoustics, can be easily verified by the results of experimental research conducted using the sound intensity technique. The presented article is an introduction to these issues and an attempt to associate the results of experimental research conducted according to this theory. The applied techniques of researching acoustic flows treated as energy phenomena, which in fact they are, refer to analyses of two basic acoustic wave vector parameters: acoustic particle 
velocity $^{1}$ - also called acoustic velocity - and sound intensity. The velocity of the acoustic particle is thousands of times smaller than the velocity of the sound $(v \ll c)$. The sound velocity $c$ in air is $342 \mathrm{~m} / \mathrm{s}$, the particle speed $v$ reaches a level around of $10^{-9} \mathrm{~m} / \mathrm{s}$.

The terms acoustic particle velocity or acoustic velocity were previously used in the descriptions of acoustic waveform flow and the classical theory of acoustics.

This vector waveform parameter has long been present in theoretical acoustics and had its own mathematical notation based on the concept of potential acoustics (Reylay, 1878), which was artificial, but successfully used to solve idealized models of acoustic field. Sound intensity is another vector parameter known for a long time, used to describe the energetic character of the wave flow It is defined as the product of the acoustic particles velocity and sound pressure $(I=v p)$. Unfortunately, due to the lack of suitable measuring transducers, verification of these two vector parameters was not possible. The possibility of direct measurement of sound intensity appeared only in the 1980s, when Fahy and Chang introduced a sound intensity probe.

These two vector parameters fully describe the dynamics of the transport of acoustic energy flowing from the source to a far field. With their help we can directly determine the acoustic power of the source, acoustic impedance and other vector parameters of acoustic flow. However, so far, these and many other parameters of an acoustic wave have been assessed mainly theoretically, while numerical approaches have often turned out to be a source of serious errors, additionally caused by the use of unjustified simplifications and linearization of equations used in calculations.

The sound field is described as a steady state disturbance, which describes the medium through which sound is transmitted. To theoretically describe the movement of a sound wave we need at least four parameters: particle speed, pressure, density and temperature. These quantities can be written in the form of average steady state values and the value of the variable (disturbances) in the following way, where the variables printed in bold are vector values [18]:

$$
\begin{array}{ll}
\text { - pressure } & P_{t o t}=P+p(r, t)[\mathrm{Pa}], \\
\text { - velocity } & U_{t o t}=U+u(r, t)[\mathrm{m} / \mathrm{s}], \\
\text { - density } & \rho_{\text {tot }}=\rho+\rho(r, t)\left[\mathrm{kg} / \mathrm{m}^{3}\right], \\
\text { - temperature } & T_{\text {tot }}=T+r(r, t)\left[{ }^{\circ} \mathrm{K}\right] .
\end{array}
$$

These parameters are functions of amplitude, place and time. Any noise problem may be described in terms of a sound source, a transmission path and a receiver, and noise control may alter any one or all of these elements. Pressure, temperature and density are scalar quantities that do not require discussion. However, explanation is required for the particle velocity $u(r, t)$ and the vector equation involving it.

\footnotetext{
${ }^{1}$ It should be noted here that the acoustic particle is a conceptual name and does not mean a physical molecule. The velocities of the acoustic particle $v$ (or $u$ ) should not be associated with the sound velocity $c$ [18].
}

The particle velocity is very important for the proper description of sound generation, but is an unnecessary parameter for the SI determination. It is a vector quantity, whose position in three-dimensional space is determined by the values of three components: $u_{x}, u_{y}, u_{z}$ (eq. 2 ). To properly describe sound generation mechanism, known values of acoustic particles velocities become essential for understanding the mechanism of converting the energy of motion into a sound effect described by the intensity of sound. The sound intensity, in turn, will be used in analysis of the results of experimental research on $3 \mathrm{D}$ acoustic flows in real environments.

For numerical modelling of the spatial distribution of an acoustic field we need to know the values of several basic parameters, such as:

1) speed of sound $c$, in air $343 \mathrm{~m} / \mathrm{s}$,

2) air density $\rho$, at sea level $1.206 \mathrm{~kg} / \mathrm{m}^{3}$,

3) acoustic impedance $\rho c$, for air $414 \mathrm{~kg} \mathrm{~s} / \mathrm{m}^{4}$,

4) temperature $T,{ }^{\circ} \mathrm{K}$,

5) ratio of specific heats $\gamma$, for air 1.40 ,

6) universal gas constant $R 8.314 \mathrm{~J} \mathrm{~mol}^{-1} \mathrm{~K}^{-1}$,

7) molecular weight $M$, for air $0.029 \mathrm{~kg} \mathrm{~mol}^{-1}$.

In the case of sound generation by vibrating structures it is still necessary to know several material source parameters like mechanical impedance $\mathrm{z}_{\mathrm{m}}=\mathrm{F} / \mathrm{u}$, value of internal material losses, complex modulus of elasticity $\mathrm{E}^{\prime}=\mathrm{E}(1+\mathrm{j} \eta)$, where $\mathrm{E}$ is the Young's modulus of elasticity, etc. Although the numerical waveform parameters obtained as a result of model calculations characterize the distribution of the acoustic field in terms of quantity, a lot of important information can also be obtained from qualitative images of the field presented graphically.

\subsection{SI-AOD Method for Practical Applications}

The sound intensity amplitude may be determined by a twomicrophone method, cross correlation transform between pressures from two microphones or as a direct measurement of pressure and acoustic particle velocity. The aforementioned advantages of the SI technique may be utilized in acoustic metrology much more effectively if the new 3D-USP miniature intensity probe is applied. The Ultimate Sound Probe - USP (Microflown [24]) is a very compact and integrated transducer that combines three orthogonally positioned particle velocity sensors and a miniature pressure microphone. The actual headsensor configuration without its cap is less than $5 \mathrm{~mm} \times 5 \mathrm{~mm} \times$ $5 \mathrm{~mm}$.

The Microflown 3D-USP, used as a scanning probe, was especially developed for measurements carried out very close to vibrating objects - the source of acoustic power. The USP effectively extends the traditional possibilities for complete sound intensity depictions of 3D energetic fields, by measuring three particle velocity vector components and the acoustic pressure. By minimizing the array distance to the sound source, we may investigate particle velocity levels in very near acoustic field conditions (called hydrodynamic region), and the power acoustic flow may now be fully described in real-life experimental conditions.

The flow of acoustic energy presented by the intensity 
streamlines (they are graphically presented as ribbons) shows the way of energy flow in an acoustic field. Showing the paths along which it is transmitted may be very useful when the necessity arises to visualise the "shape of noise" radiated by vibrating mechanical structures (machines, vibrating heterogeneous plates, devices, etc.) and can show their activity also in limited spaces. This is a form of qualitative analysis for stationary fields which consists in a complex evaluation of the paths along which the acoustic energy of a radiating source is transported. Additionally, the shapes of intensity isosurfaces in three-dimensional space were presented to bring a new insight into the interpretation of the results of measuring the radiation of acoustic sources, acting as sources of noise radiated by vibrating surfaces, e.g. walls, ceilings and floors of living quarters on ships.

Based on the research with intensity technique and using selected AI methods, the tests demonstrate vector space distribution of the acoustic field in the area adjacent to the ship partitions. This provides significantly new information related to energetic acoustic fields in the vicinity of vibrating partitions which, in turn, affect the noise distribution inside the cabin.

The ship partitions, characterised by high structural heterogeneity (partitions made of panels of sandwich structure, walls with windows and doors), are surfaces of heterogeneous distribution of vibrations, and the acoustic power radiated by such a surface source may be determined experimentally. In this case, the sound intensity technique is a very useful tool in seeking noise abatement solutions on ships.

The application of new measurement techniques described in this paper, different from the methods used in classical acoustics, will allow acquiring credible technical information, which in turn will make it possible to:

1) indicate dominating noise sources on a ship,

2) identify noise generation mechanism by respective ship sources, and separate energetic share for structural and air noises,

3) indicate, based on theoretical knowledge supported by experimental research results, propagation paths of vibro-acoustical effects (vibrations and noise) in ship structure through which the energy will penetrate into ship cabins,

4) indicate effective and economical technical methods - construction-wise and/or technological - of elimination or limitation of excessive but permissible vibration and noise level standards that can occur on a newly built ship,

5) indicate and justify differences, which have to be considered in the description of noise level generated on ship and methods of its anticipation and limitation, if different materials are going to be used for the construction of the hull and equipment elements (steel, aluminium, fibreglass or carbon fibre composites),

6) make the available ship-noise-level prognosis methods more efficient, by using the limited technical data already available at the early ship design stages.

\subsection{Acoustical Imaging and Orthogonal Decomposition of the 3D Acoustic Field}

Acoustical imaging (AI) from vector intensity measurements allows us to analyse various wave events occurring in real acoustic fields, theoretically described by VST. We can record images of the effects of changes in the field structure on the obstacles affected by the acoustic wave, the effects of reflections, refraction and dispersion, wave reactions in interiors of rooms and flows in pipe installations. The registration of flow disturbances reveals the mechanism of the formation of hydrodynamic and aerodynamic reactions, which in the process of laminar flows become turbulent and cause noise.

The visualisation methods for graphic descriptions of the effects of fluid flow have been advanced over the past several years and are described in detail [25-27]. Many of the techniques used in computer graphics flow visualisation have been adapted from traditional methods used in wind and water tunnels. Scientific visualisation is the use of computer graphics to create visual images which aid the understanding of this often immense data set. The visualisation system, by serving a dual role as a provider of exploration and exposition capabilities, has become indispensable for the analysis of Computational Fluid Dynamics (CFD) results [28]. Experimental flow visualization of waves is inspired to know the mechanisms of acoustic turbulent flows can bring some additional knowledge about acoustic vortex flows.

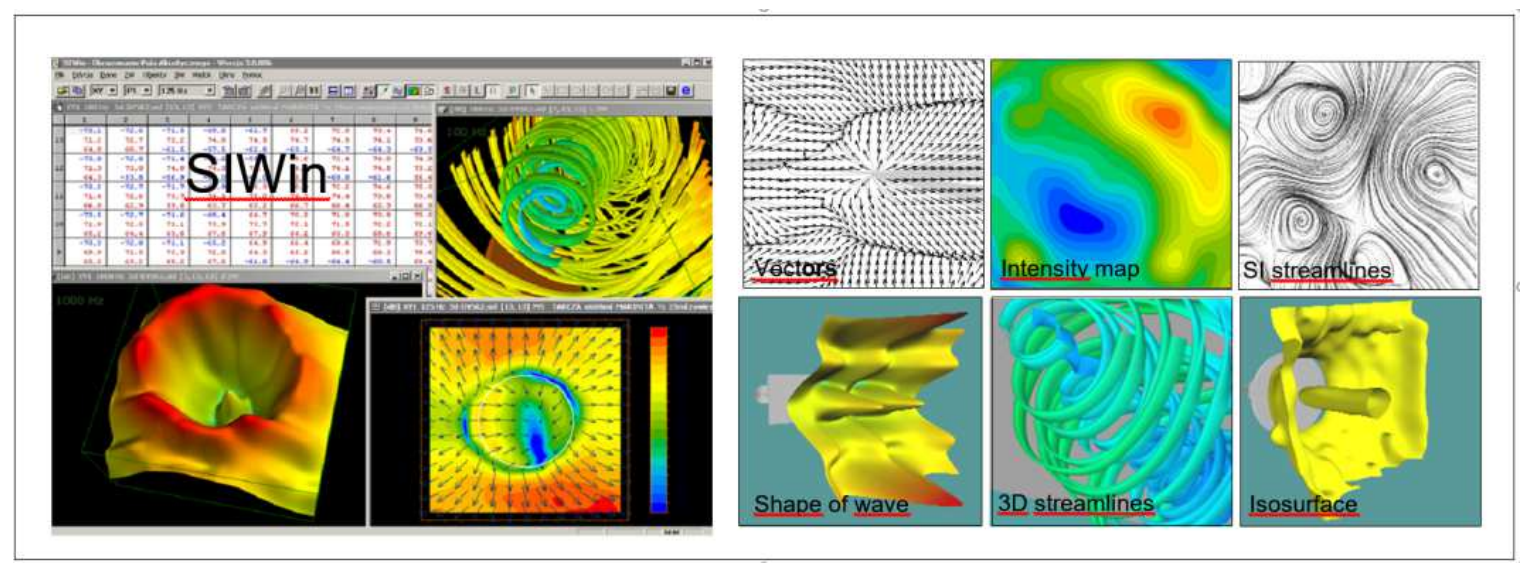

Figure 2. Implementation of the SIWin post-processing program for imaging energy distribution of acoustic vector fields. 
The author has described the visualization methods in acoustic flow fields and showed how these methods may assist scientists to gain understanding of complex acoustic energy flow in a real-life field. Acoustical images will be created using the original SIWin program (Figure 2) and the animations of the recorded wave movement can be presented with AniviewWnd [29]. A graphical method will be presented to determine the real acoustic wave distribution in 2D and 3D flow field. Visualized research results are shown in the form of intensity streamlines in space and as a shape of floating acoustic wave and intensity isosurface in three-dimensional space (Figure 3), which is unavailable in conventional acoustics metrology. In traditional acoustic metrology, the analysis of acoustic fields refers only to the distribution of pressure levels (scalar variable), while in a real acoustic field both scalar (acoustic pressure) and vector (the acoustic particle velocity) effects are closely related.

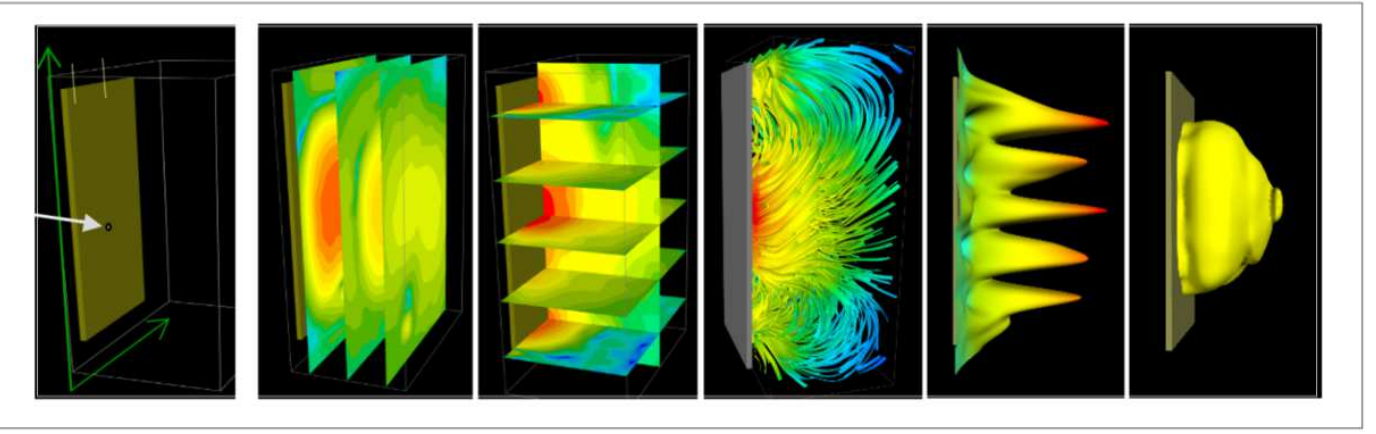

Figure 3. Various visualizations of the acoustic emission of a vibrating plate: 2D maps of the intensity distribution, intensity streamlines.

It follows from the experimental works conducted by the author that the visualization of acoustic intensity streamlines enables a full interpretation of vector phenomena in an acoustic flow field, which can be confirmed by a few examples from our previous work with AI-SI method [27, 33, 51]. Fragments of the results of the research on noise sources in ship's rooms are presented in Figure 4. Images of diffraction and scattering on obstacles which show vortices and rotations phenomena associated with acoustic energy flow within a elastic medium can enrich the knowledge of the mechanism of acoustic energy flux. As we cannot obtain this information from traditional measurements of acoustic pressure, sound intensity is a useful tool for acoustical imaging, a previously unknown method of describing wave motion in experimental acoustics.

Thanks to the sound intensity measurement and the vector study of the movement of acoustic waves, we can visualize the dynamic phenomena caused by the flow of an acoustic wave in the area of obstacles in the way of wave flow. Experimental visualization of the waveform is inspired by understanding the mechanism of generation of disturbed acoustic flows and supplements the knowledge of the vortex sound theory (VST).

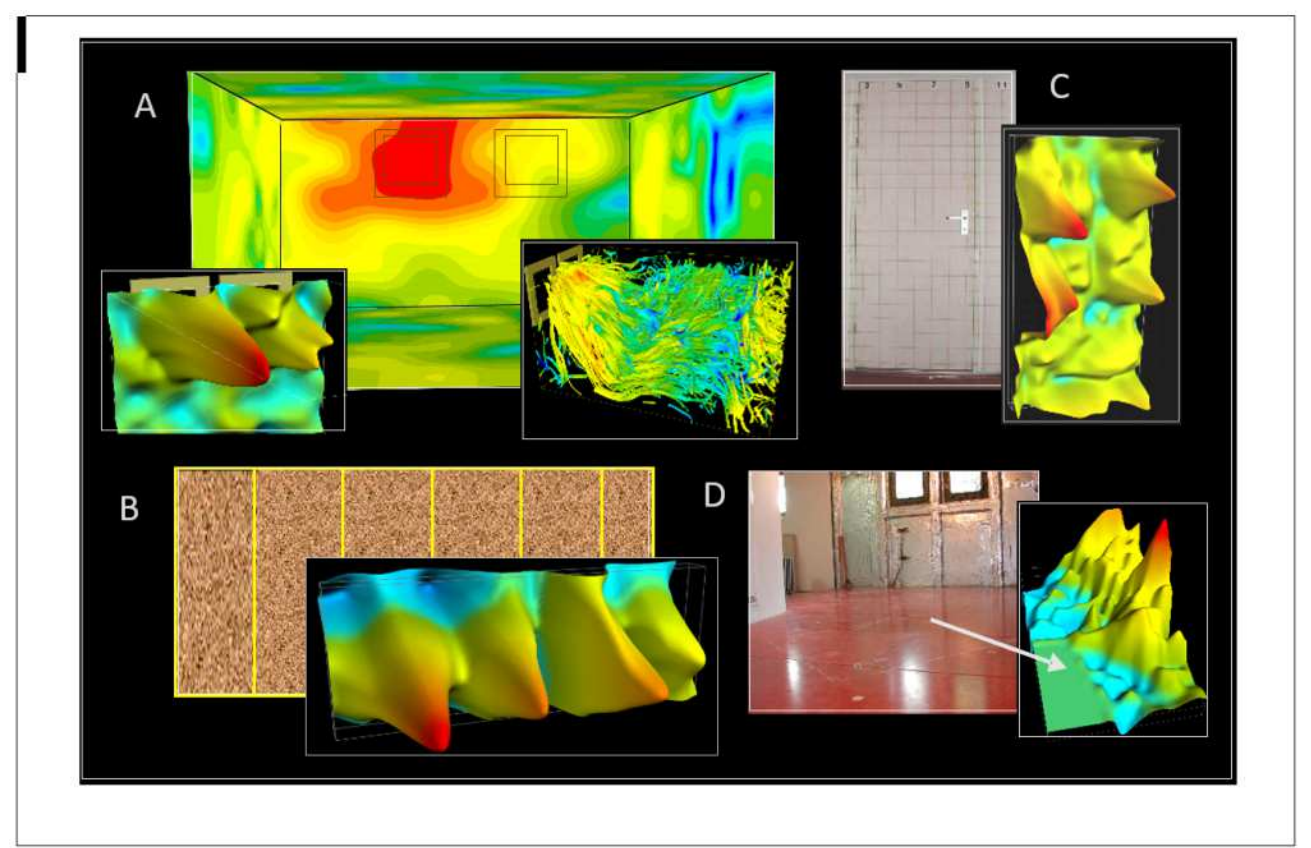

Figure 4. Some results of research by SI-AOD method of the ship's cabin noise emission: A - local sources of noise in cabin interior, B - noise penetration through the wall, $C$ - sound insulation of cabin doors, $D$ - noise radiation through the floor. 
AI analysis, with three-dimensional distribution of vector values represented by sound intensity, may be a convenient tool in visualizing disturbed acoustic fields. For this we recommend using our tool called Acoustic Orthogonal Distribution (AOD). Spatial SI vectors in 3D real field are divided into three orthogonal component planes in the Cartesian system: $x y, x z$ and $y z$. After decomposition of the field into 2D images, it turns out that orthogonal components of chaotic wave motion depict deterministic features of chaos.
By combining the commonly used frequency decomposition of acoustic field in the range of standard octave frequencies (in band $1 / 3$ or $1 / 12$ octaves) with the decomposition of AOD, we get surprisingly regular images of seemingly chaotic 3D field structures (Figure 5). Extremely regular deterministic chaotic attractors of wave motion are visible: images of vortices, sources and sinks, rotations, saddles and bifurcating lines. It turns out that the AOD method used in experimental studies creates new possibilities of describing wave motion.

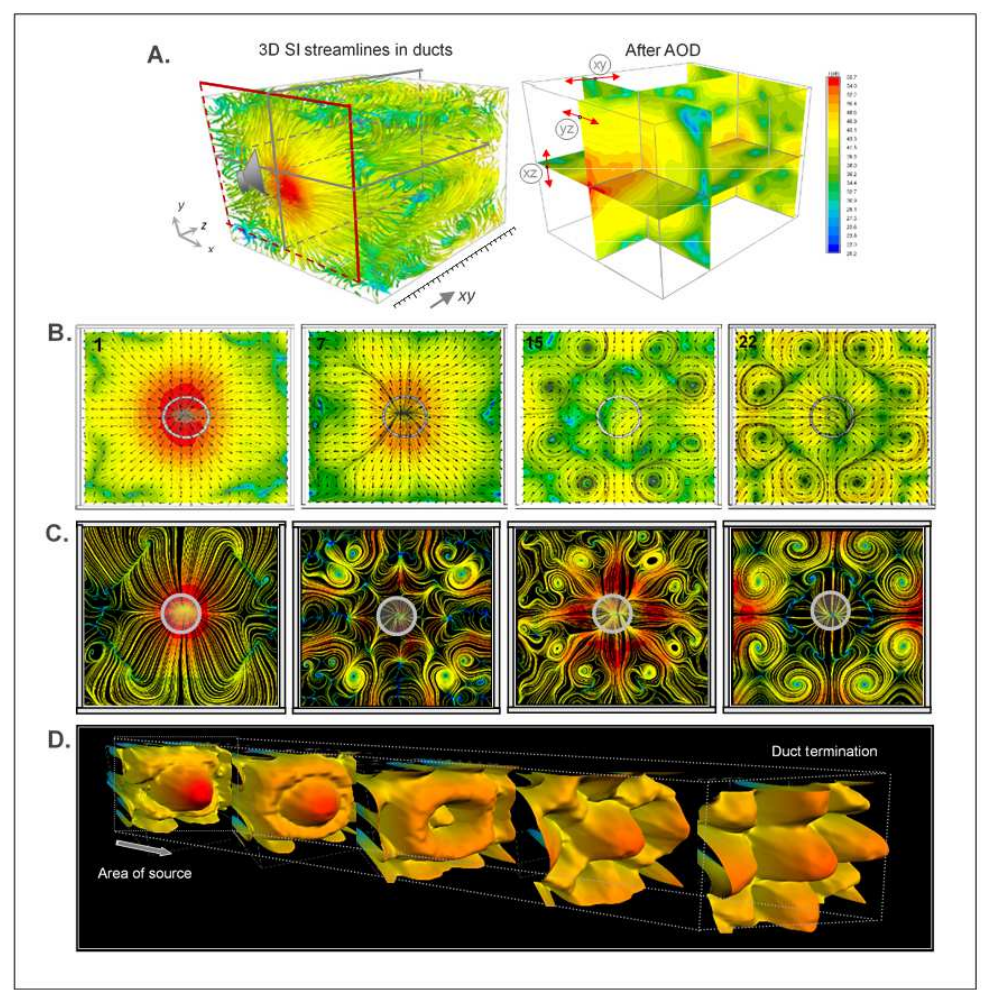

Figure 5. Orthogonal decomposition of the acoustic field in a square waveguide with an open end, frequency $1540 \mathrm{~Hz}: \mathrm{A}$ - cutting planes, B - images of subsequent xy sections, vectors map, $C$ - images of subsequent xy sections, streamlines, $D$ - sequential $3 D$ images of wave shapes in the interior.

The visualization of the effects of scattering, reflection and refraction for various cases of vortically disturbed real flow fields, theoretically described by the VST, plays an important role in broadening the knowledge of the mechanics of sound and noise generation. Presents imaging of diffraction and scattering on obstacles which show vortices and rotations phenomena associate with acoustic energy flow within a spring medium. SI-AOD acoustical imaging showing in a three-dimensional field the shape of a traveling acoustic wave, the paths of the wave (streamlines) or isosurfaces of intensity - all images built on a single database - enables a full interpretation of vector phenomena in an acoustic flow field. SI-AOD analysis can be a useful form of acoustical imaging, a previously unknown method of describing wave motion in experimental acoustics. The experimental visualization of the structure of the acoustic field is inspired by learning about the mechanisms of the formation of acoustic turbulent flows and can be used to expand the knowledge of VST. It should help experts to draw the right conclusions in future research on noise reduction in industrial equipment.

\subsection{Laser Methods in Anemometry and Acoustical Imaging}

In scientific research and in industrial applications, fluid flows are often utilized to serve diverse functions. The associated physical processes constantly require accurate quantifications and optimizations, especially for flow dynamics. Obviously, each type of flow has specific dynamic parameters. Turbulence is the final state of disturbed flows, which in mathematical descriptions is chaos (Lifszyc, Landau, Kolmogorov, Lyapunov). Today we know that the phenomenon of chaos has deterministic features [30]. Parameters of deterministic chaos described in the entire phase space by combined functions with vector characteristics are used to visualize turbulent flows in the form of characteristic attractors: sources, sinks, vortices, saddles and bifurcation lines [31]. Extensive descriptions of deterministic turbulent flows used in fluid mechanics concern mainly flows in liquids and gases. In the study of acoustic 
flow, they are of great importance in the new description of the mechanism of sound formation [32, 33].

Although nowadays the method of computational fluid dynamics (CFD) has been found to be of wide applications evaluating complex flows and improving the flow processes, its general reliability and applicability still need to be enhanced, especially through experimental validations [22]. Moreover, the CFD method is unable to replace measurement experiments which should be taken into account in flow tests and optimization of related flow processes.

Experimental flow measurement, an essential means to study and improve engineering flows and flow processes, has been an advanced technique since laser anemometry was used for this purpose. Contrary to the traditional methods of mechanical probes, the laser method obviously provides the most effective and accurate tools for non-intrusive flow measurements.

Nowadays, laser methods used for flow measurement have become very common, mainly due to many applications in industry [34-38]. Optical measurement methods have many advantages over invasive methods. The physical transducer is not introduced into the flow field and therefore does not cause any field deformation.

The most commonly laser methods for flow measurements are the Particle Image Velocimetry (PIV) [39] and the Laser Doppler Velocimetry (LDV) [40], also known as the Laser Doppler Anemometry (LDA). Both techniques have also been adapted to the measurement of acoustic flow fields [41, 42], but so far they have been limited to the measurement of flows with low forcing time-stable velocities (mean flow).

These techniques can be seen as complementary because combined, LDV and PIV take advantage of space (PIV) temporal resolution (LDV). While the PIV method is suitable to quantitatively present the flow distribution, the LDV method is mostly applied to accurately diagnose and quantify all types of flows. Thus, both of these measurement techniques are well suited for capturing complex flow in:
1) turbulence tests,

2) 3D-flow structure visualization,

3) full 3D-vortex analysis,

4) flow-structure-interaction.

Laser measurement systems are used to explore and map different types of flows. The ability of the signal processor to extract accurate velocity information is crucial for getting the needed information about the dynamic parameters of acoustic flow. Advanced laser and computer technologies have significantly broadened knowledge about the dynamics of real-live flows. Only few investigations and developments have been conducted with regards to acoustic laser anemometry. As has been perceived for a long time, it clearly lacks a supportive reference for PIV/LDV acoustical user in practical applications.

\subsection{Adapting Laser Techniques to Acoustics}

Laser anemometry allows the non-intrusive instantaneous measurement of a field of vectors. The nonintrusive property of laser measurement methods represents the greatest advantage against other methods using sensors for flow measurements. LDV and PIV have been used for the first time for measurements in fluid mechanics and their application to acoustic flow is rather rare compared to SI flow studies. It is now widely used to measure fluid dynamics in many acoustic events, although the advantages of acoustic laser anemometry are unquestionable [43-44]. Laser methods can be also adapted to provide an instantaneous flow and acoustic particle velocity and sound intensity mapping with no disturbance of the sound field (Figure 6). Acoustic flow velocity measurements by means of PIV/LDV methods are based on random sampling of velocity events occurring when the seeded particles pass through the measurement volume. The available velocity sampling rate depends on particle concentration, particle size, flow velocity and other flow and optical parameters.

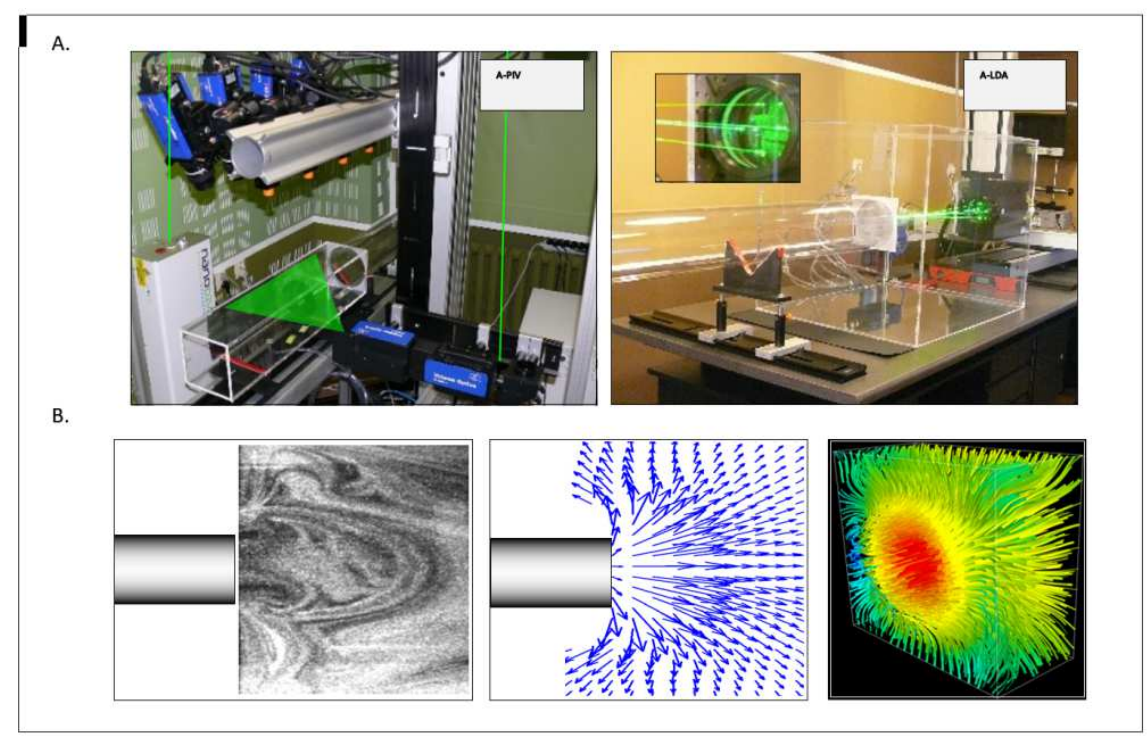

Figure 6. Two laser measuring stands for testing of vector structures (A) and example pictures of acoustic flow outside end of open duct; particle movement and the SI distribution at the outlet of an open ended duct (B). 
Sound measurements are better in some respects compared to classical fluid mechanic measurements. The downside is that acoustic velocities and displacements are generally quite small (for instance at $1000 \mathrm{~Hz}$, for an acoustic level of 100 $\mathrm{dB}$ SPL the velocity amplitude is about $5 \mathrm{~mm} / \mathrm{s}$ and the displacement amplitude about $10^{-6} \mathrm{~m}$ ) and vary rapidly in time.

The primary objective of our research is to develop techniques and tools for visualizing complex acoustic flows as Acoustic-PIV and Acoustic-LDA. This makes A-PIV/ALDV methods widely used in the measurement of internal acoustic flows, such as flows in ducts and turbo-machines.

\section{General Description of Noise Generation by Ship's Machinery}

\subsection{Shipboard Machinery as Sources of Noise}

Any noise problem can be described in terms of sound source, transmission path and receiver, while noise control can consist of changing one or all of these elements. The most effective outcome of reducing the noise of a machine or device is vibro-acoustic modification of a noise source, i.e. application of active noise abatement method. Active acoustic protection is not always possible. Practice shows that it is easier and cheaper to modify transmission paths or use engineering passive noise abatement method to reduce the effectiveness of noise radiation into the environment. It is possible to consider various options for control and finally determine the cost effectiveness of the various options.

Ship engines release energy into the air and the steel structure of the ship through their foundations. The transmission of vibroacoustic energy through the ship's structure consists of a continuous transformation of mechanical energy (structure-borne noise) into acoustic energy (airborne noise). Mechanical energy is effectively transferred inside the structure, due to its relative flexibility and low damping, reaching zones quite distant from the engine room.

The vibrations of floors, walls and ceilings, in turn, generate noise inside rooms occupied by passengers or crew. On the other hand, airborne noise from engines can easily spread within the ship through piping of HVAC systems. The next noise path is a sequence of piping systems (flue gas ducts, hydraulic automation, fuel transport, etc.), which run in the whole volume of the hull, from the engine room to the upper decks of the ship.

An analysis of noise transmission on a ship is complicated due to several transmission paths connecting sources and potential receivers. These paths may include portions in the structure and in the air, with several possible conversions from structure- to air-borne noise and vice-versa. Fortunately, from the point of view of a regulatory action, the receiving position as well as the source and the transmission paths are placed on the ship, so that the whole process can be controlled at the design and verification stages by the same body.

\subsection{Mechanism of Noise Transmission to Ship Interiors}

Sound, which becomes noise at high amplitudes, is an energy phenomenon caused by the oscillating movement of environmental particles. Its generation can be the result of various hydro- and aerodynamic waveforms of a turbulent nature, like those caused by aeroacoustic effects or natural phenomena, impacts, explosions or implosions. Industrial noise is usually associated with vibration of mechanical structures. For example, intense noise can be generated by machine casings, industrial installations, construction machinery, rocket engines, aircraft and watercraft. Creaking and squeaking noises, roar of engines and whistles from HVAC are the main reported causes of annoyance. Investigation results demonstrate the prominent role of acoustics in respect of shipboard comfort.

If an enclosed space is located in the field generated by noise sources, acoustic energy limited by the insulating properties of the enclosure material will be transmitted to the interior through the housing. The mechanism of energy transmission will depend on the local acoustic impedances of individual fragments of the casing. These fragments can be treated as local sources of noise generating a specific sound power through the surface $\mathrm{s}\left(\mathrm{N}_{\mathrm{ac}}=\mathrm{I} \cdot \mathrm{s}\right)$. The sum of the power of local sources is the total value of acoustic energy (noise) transferred to the interior, but most often this energy effect of noise is measured and reported as the sound pressure level $\left(\mathrm{SPL}\right.$ or $\mathrm{L}_{\mathrm{p}}$ in $\left.\mathrm{dB}\right)$.

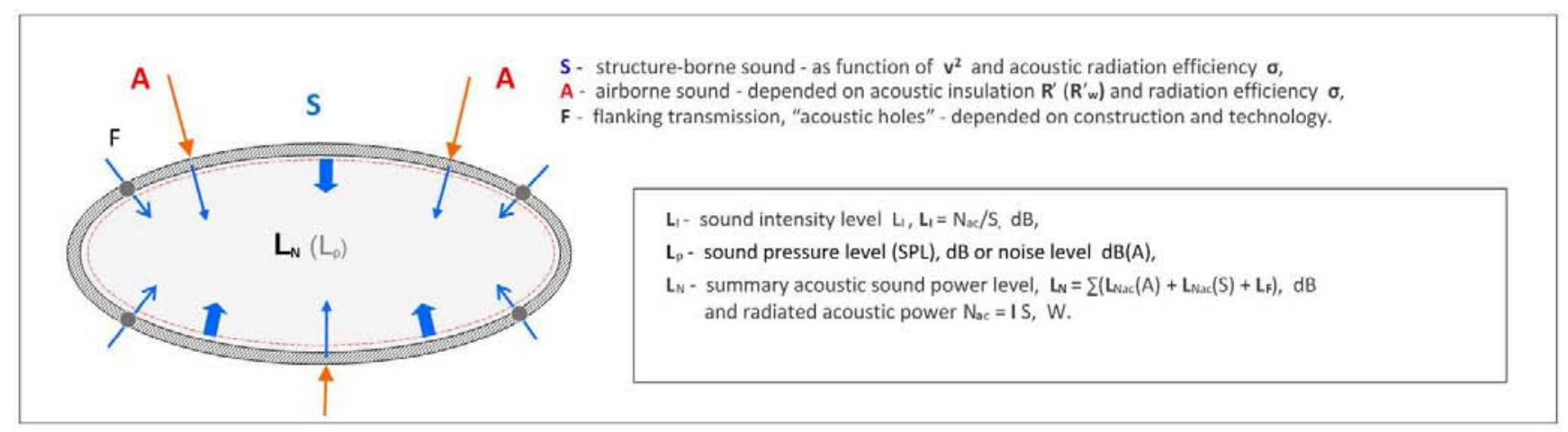

Figure 7. Holistic description of the mechanism of noise (vibro-acoustic energy) transmission to ship interiors. 
The resultant acoustic field in each interior comes from simultaneous action of many independent local sources created by structure-borne sounds (S), airborne sounds (A) and sounds created by radiation of acoustic energy generated by surface vibrations of structures surrounding the interior (Figure 7). Some airborne sounds pass directly into the interior through technological leaks as so-called flanking transmission (F). Due to the non-linear character of amplitude-phase relations and the simultaneous interaction of acoustic waves, very complex sound field structures are created inside. These phenomena are difficult to describe with a simple mathematical model. However, this holistic mechanism of noise generation in confined spaces can be studied and experimentally controlled using the measurements of acoustic parameters, shown in Figure 7. A detailed methodology of research into ship interior noise through the use of particular parameters will be presented in the second part of the publication.
The noise inside ship's compartments is always associated with the simultaneous action of structure-borne and airborne noise carried by the ship's hull structure, and sounds originated through radiation by excited local structures (Figure 8). Synergetic joint action of these various exterior and interior sources makes the resulting acoustical field the holistic phenomenon which cannot be reduced to the sum of its components. Although structural sounds are assumed to dominate shipboard noise, the relationship between structural and airborne sounds can vary (see Figure 8A). The noise hazard on a ship decreases with the distance from the main noise sources, commonly concentrated in the stern region (propulsion noise) and the engine room (mechanical noise). The further away from these sources the room is installed, the lower noise level in the interior. Usually the noise level on successive decks decreases by 3 to $5 \mathrm{~dB}$ on deck, except for upper decks in the areas of the funnel.

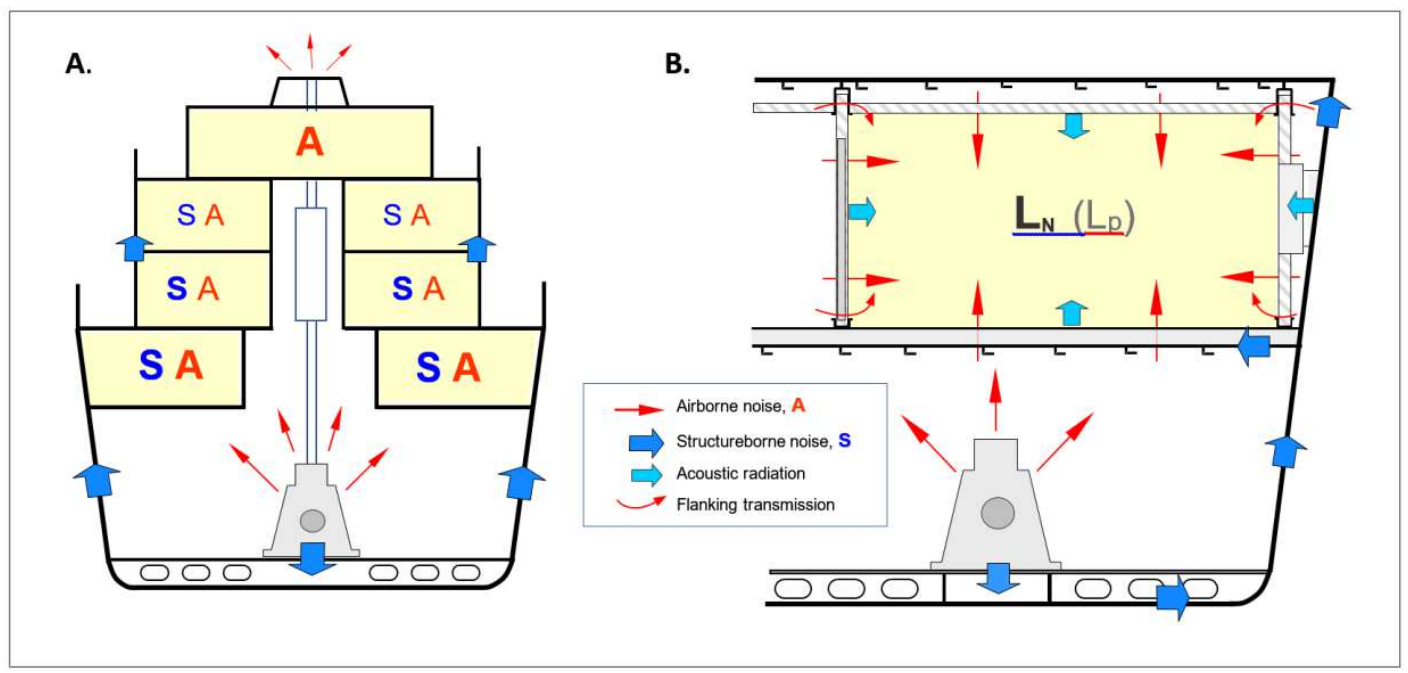

Figure 8. Vibro-acoustic model of noise transport in ship's structure divided into structure-borne and airborne sounds, flanking transmission and acoustic radiation (A) and paths of acoustic energy (noise) transport into the ship's cabin (B).

The measurements of noise parameters presented in the article, specified in Figure 7, will be carried out using techniques that differ significantly from traditional measurements. The scope of the proposed research is very wide and is concerned with vibroacoustics problems and fluid mechanics together with aerodynamics - areas of knowledge closely related to the theory of acoustic flows. Therefore, the innovativeness of the proposed research topic is also important for general acoustic metrology, because it is concerned with areas of acoustical knowledge so far poorly recognized experimentally. The proposed topic of experimental research into acoustically disturbed flow fields still evokes great interest of scientific research institutions, which direct the acquired knowledge to practical use in the dynamics of rotating machines and turbo-engines, flow motors, pumps and fans, hydrodynamic flows in tubular installations of the chemical industry, in ventilation and airconditioning systems, in cooling towers, silencer constructions, etc.

Two measuring techniques prove to be extremely useful and effective in shipbuilding applications: the sound intensity technique (SI) and the laser anemometry technique (PIV and LDA), which treat an acoustic wave asa stream of energy moving in the real field. The parametrised energy of movement has vector characteristics and, for an acoustic wave, its movement is represented by acoustic particle velocity and sound intensity. These two parameters with vector characteristics allow for an image-based analysis of the dynamics of acoustic wave motion and, on the basis of the estimation of the field distribution, effectively find sources of noise. In classical acoustics based on measurements of acoustic pressure, a scalar waveform parameter such as visualization was not possible.

In the presented publication we want to demonstrate advantages of vector noise analyses in research related to ship's acoustics and, when interpreting the results, show that 
vector distributions of acoustic fields in ship's rooms described by these techniques share many features with hydro- and aerodynamic flows known from mechanics. These links between acoustics and mechanics allow acoustics to benefit from the rich knowledge of fluid mechanics about hydrodynamic flows and make it easier for acoustics to draw correct conclusions from research into the energy effects of acoustic flows.

\subsection{Flows in Installation Ducts as a Noise Source}

The unconventional research techniques presented in the paper on the reduction of ship's vibrations and noises also include the study of noise hazards from ship's piping systems, with ducts of heating, ventilation and airconditioning (HVAC) systems. Many such installations exist in the ship's engine room, on decks (especially on tankers) and in superstructure spaces. They are often a serious source of structure-borne and airborne sounds traveling in the ship's hull structure. In the living quarters on ships, the noise of ventilation and air conditioning systems is often the main cause of exceeding permissible noise levels. The level of installation noise is the most annoying for the crew and passengers when ships are waiting on the roadstead for port entry and while in port.

The main noise emission sources on ships are the propulsion machinery located in the ship's engine room and the ship's propeller. There are also secondary sources of noise, such as: auxiliary machinery, hydraulic systems, different types of pumps, and the effects of the sea, wind, etc. Forced ventilation and other HVAC systems are assessed as the third major source of ship noise, originating from: fan itself and its motor, air ducts, diffusers and ventilation regulators [45].

The ranking of the ship's piping systems in terms of noise nuisance for crew and passengers can be as follows:

1) Heating, ventilation and air conditioning systems,

2) Diesel engine exhaust systems, ventilation grilles, exhaust gas stacks,

3) Inlets and discharges of HVAC systems,

4) Automation systems for hydraulic control of such devices as jet controllers,

5) Steering machines, rolling stabilization systems,

6) Fuel transport pipes and pipelines on tankers,

7) Ballast installations.

The majority of flows in piping systems in nature and in engineering applications are turbulent. Turbulent flow fields are three dimensional, chaotic, diffusive, dissipative, and random. These flows are characterized by velocity fluctuations in all directions with infinite number of scales. Exact analytical solutions of Navier-Stokes equations which are statements of the conservation of mass, momentum, and energy for turbulent flows are not currently possible since these equations are elliptic, non-linear, and coupled [46]. Although direct numerical simulation (DNS) of turbulent flows gives better results, it is not currently practical due to significant computational resources required. So far, DNS approach has only been applied for a limited class of simple low Reynolds number applications.

Presently, turbulence modeling based on ReynoldsAveraged Navier Stokes equations is the most common and practical approach for turbulence simulation. RANS are timeaveraged modified Navier-Stokes equations, and turbulence models are semi-empirical mathematical relations used to predict the general effect of turbulence. The method most commonly used to model acoustic flows is a proposal for the description of the noise source given by Ffocs WilliamsHawkings (FWH) model, [47, 48]. This model is the most similar to the description of the energy effects caused by an acoustic flow in real conditions and is consistent with the rules of VST theory.

The objective of turbulence modeling is to develop equations that will predict the time-averaged velocity, pressure, and temperature fields without calculating the complete turbulent flow pattern as a function of time. Unfortunately, there is no single universally accepted turbulence model that works for all flows and all regimes. Therefore, users have to use engineering judgment to choose from a number of different alternatives since the accuracy and effectiveness of each model varies depending on the application. This justifies the extremely important role of experimental research, the results of which should be treated as part of the validation of numerical models. If the results of control tests are to provide the authors of theoretical numerical models with an acceptable standard of correctness of the solvers used, they must be convinced that the experimental research techniques take into account the current level of theoretical knowledge of the phenomenon under investigation and that the measurements guarantee metrological correctness.

Unconventional research techniques presented in the paper on the reduction of vibrations and noise on ships also include research on noise hazards caused by ducts, and pipes appear to meet these requirements. Two innovative measuring techniques will be used in the presented studies: intensity method (SI-AOD) and laser image anemometry (A-PIV/ALDA). The new methods consist in recording energy parameters of wave motion and spatial visualization of structures of disturbed acoustic flows in ducts, which are the cause of excessive vibration and noise in the environment. The proposed research techniques, together with the use of new measuring equipment, will make it easier for designers and constructors to choose effective technical methods to reduce noise of pipe installations on ships.

SI-AOD method of investigation in ducts gives a physical picture of sound waves in any duct mode. However, an acoustic field is extremely complex because pressures in nonpropagating (cut-off) modes cooperate with the particle velocities in propagating modes, and vice versa. Above a critical frequency, the flow becomes turbulent (cut-on structures) with a high noise level. Measurements in cylindrical ducts demonstrate also the cut-on phenomenon and the effect of reflection at the open end [49]. The experimental results can also show the effects of axial and swirling flow (e.g. see Figure 9). 
The aim of our experimental study (see next publication by this author) will be to obtain information on low Mach number flows in ducts in order to enhance physical understanding of these flows and to provide well documented test cases to validate theoretical CFD/FSI/CAA models that still are imperfect. In our investigations, sound intensity generation in a hard-walled duct with acoustic flow (no mean-flow) will be treated experimentally and showed graphically in a manner very similar to that described by
POD and DMD calculation methods. Along the duct, sound energy is carried in various modes, whose shapes will be examined. Visualization illustrates the vortex flow as sound intensity streamlines and will graphically explain diffraction and scattering phenomena occurring inside the duct and in the termination area. Further research on the energy interaction phenomena between axial and radial modes inside ducts will aim at more realistic engineering applications.

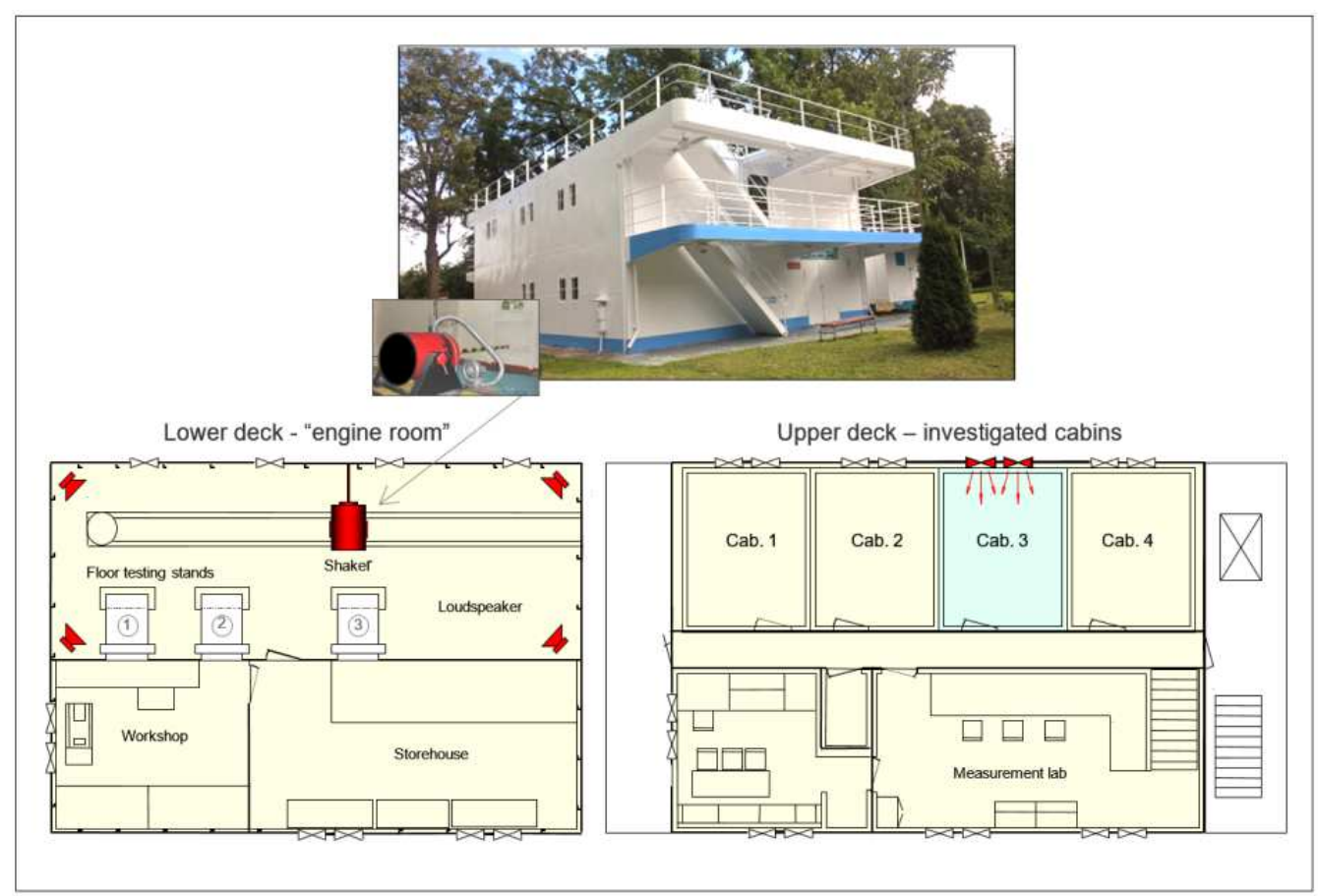

Figure 9. Mock-up superstructure built at the university premises as a laboratory for vibroacoustic testing of full-size cabins with furniture.

\subsection{The Function of Experimental Research in Examining Prototype Structures}

The modern process of designing new industrial products relies on numerical calculation techniques and simulation of the results. This greatly simplifies the design technique and makes it easier to start mass production of already comprehensively optimised product. Modern engineering, however, depends on experimental research, a necessary element for the verification of computer techniques. Technical parameters of a product must be checked and confirmed by experimental measurements. It is also the second stage of the product improvement cycle and documentary confirmation of its quality.

Design of sea-going ships goes in line with the current strategy adopted in contemporary industrial product design. Shipbuilding products are also constantly optimized technically. Recently, the high technical quality of commercial and passenger ships is necessitated by expected improvement of working conditions of the crew and the comfort of passengers. One of the attributes of comfort is low noise level. Ships with low noise levels are also environmentally friendly for the marine fauna and flora. Quiet ships have become a social requirement and force shipbuilders and owners to meet the classification requirements for Quiet Ships.

It is clear that more effective solutions should be sought in developmental research into silent ships, for which noise levels are becoming a primary technical parameter. To this end, our team of academic research workers is trying to use the many years of experience they have gained in research on ship vibroacoustic protection conducted in specialized laboratories and directly on ships. It should be pointed out that the research is not conducted using traditional methods and its effectiveness has been tested in shipbuilding practice.

The proposed test method based on the SI_AOD technique in combination with a graphical presentation of the distribution of acoustic intensity or power (AI) is performed using our own post-processing software (SIWin). The results of the research give a full picture of the energy effects caused by noise and allow to optimize the protection against noise on ships. For greater certainty, the choice of an optimal solution for protecting prototype rooms is usually supported by laboratory tests.

The measurements described were carried out in real size crew cabins built on the second deck of full scale mock-up superstructure (Figure 10). The superstructure was built on 
shore as a ship acoustic laboratory to carry out dynamic research in conditions close to the real ones. For classical investigations we use standard sound signals such as white and pink noise. To simulate the excitation from a ship engine (airborne and structure-borne noise), an electrodynamic exciter and high power loudspeakers are used for acting on the steel hull under the investigated cabins. The noise present in the ship accommodation comes mainly from structural sounds, generated by an electomagnetic shaker acting on the superstructure model, although air noise from the "ship engine room" of the model is also taken into account. In order to bring the test conditions closer to the actual conditions on ships, the forcing signals used in the tests come from direct recordings made on ships in service.

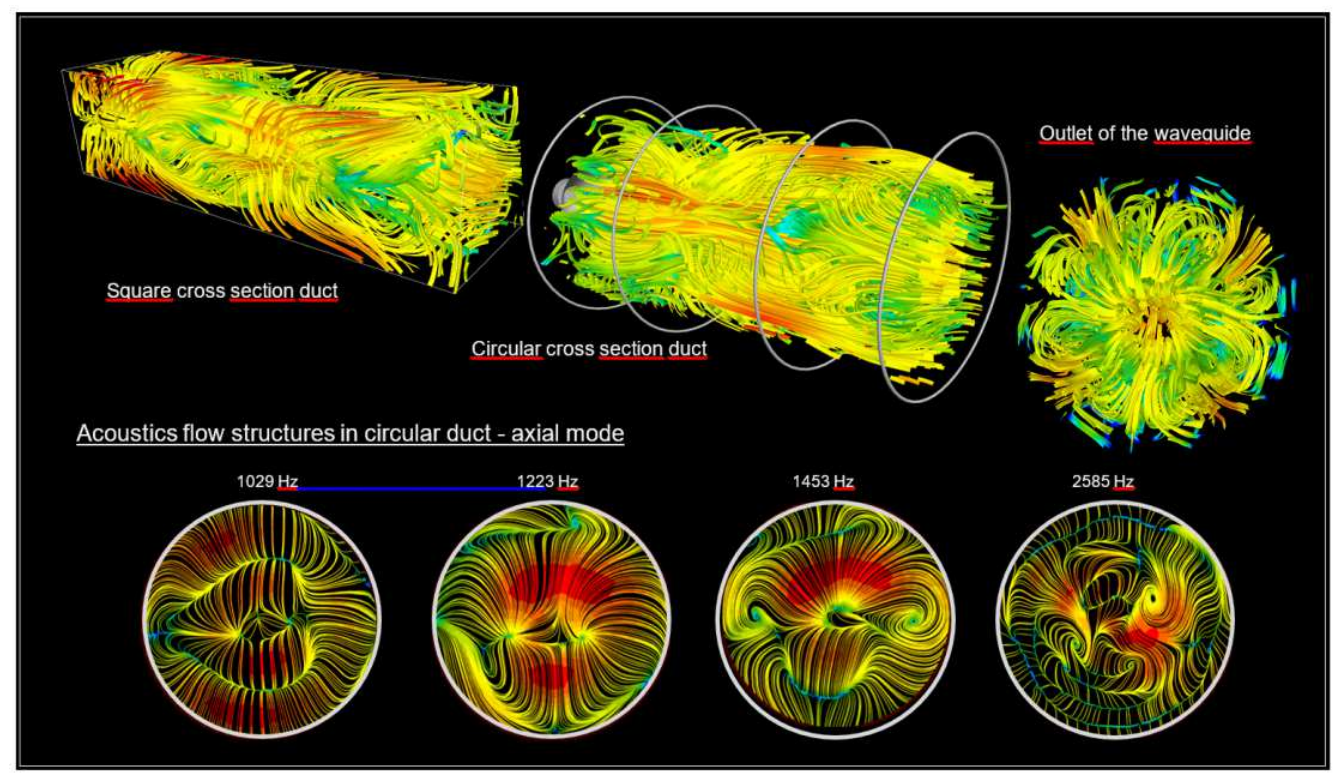

Figure 10. Spatial structures of the acoustic field in waveguides recorded by laser anemometry and images of cross-sectional sound intensity streamlines fields above cut-off frequencies ( $848 \mathrm{~Hz}$ ) after AOD decomposition.

\section{Prediction of Vibrations and Noise on Ships}

Typical sources of vibration and noise in a ship are diesel engine, propellers, gear boxes, pumps, air compressors, electric engines, generators and HVAC installations. The vibration energy is transmitted by the structure through longitudinal, torsional and flexural waves.

Structural noise can be generated in each machinery unit, either directly, by the coupling of vibration machinery over the structure, or indirectly, through the impact of airborne noise in the unit generating structural vibration. Radiated noise is mostly caused by flexural waves, but they are predominant in the structure. It consists, briefly, in transforming the energy input of the noise source or vibration in the vibrational waves will be damped by the structure, through structural discontinuities, and thereafter processed again in air-borne sound through vibration of structural elements compartment receptor. The conditions to carry out measurements on machine foundations are provided following international standards set by government agencies specialized in the construction of ships and platforms. The fulfillment of these standards will depend on the classification society supervising the construction and the regulations of the ship's flag state.

In order to predict and limit noise levels which may occur in compartments of a ship under design, we need to know the mechanism of radiating acoustic energy from vibrating mechanical structures. It is also necessary to know acoustic properties of the ship's equipment materials influencing the absorption of acoustic energy, i.e. the possibility of shaping the characteristics of acoustic impedance, acoustic absorption, viscous and thermal effects of material losses affecting noise reduction. It is required to supplement the existing knowledge of generation and transport of acoustic energy in a gas medium, especially in the air. It is first and foremost about a deeper analysis of the genesis of wave motion, which is born when environmental particles are stimulated to vibrate and then move in the medium on the flow of spring energy.

For the shipbuilding industry, reduction of vibration and noise on ships under construction to a level set out in classification society regulations and standards is a prerequisite for the ship to be put into service. The methods used to calculate sound emissions and propagation should comply with the latest directives and standards. Access to accurate component data with respect to material, geometry and boundary conditions is essential to achieve correct calculations. Although a simulation calculation model is created from the ship's structural steelwork plans or detailed drawings of structural elements, the exact descriptions of the ship's propulsion systems and the power values of the installed propulsion and equipment are not yet known at the preliminary design stage.

The designer can find out about possible risks of 
exceeding the acceptable standards for a newly built ship at an early stage of the design work, as long as he has access to the results of the forecast calculations. The accuracy of forecast calculations depends mainly on the chosen tool, but also on the access to correct material and structural data of the designed ship's hull structural elements. Numerical programs used for ship sound level analysis and mapping have become increasingly refined over the years but still incorporate the basic elements of architectural acoustics. It is important to know the mechanism of noise generation and mutual sources of noise described on the basis of the latest developments in modelling noise on board ships.

It is suggested that a similar approach be attempted when trying to obtain agreement between noise predictions and measurements when working on new, more effective research methods.

Several promising programs have been written that employ finite-element analysis, boundary-element analysis, and statistical-energy analysis techniques to predict airborne and structure-borne noise generated by ship sources. For prediction of ship noise, the finite element method (FEM) and the boundary elements method (BEM) are usually used to simulate many different technical scenarios. In structural dynamics and acoustics, these two techniques are primarily important for the lower frequency ranges. Problems in the higher frequencies are addressed by statistical methods such as statistical energy analysis (SEA) [50].

The SEA method was introduced in the 1960s as a computational tool to predict the broadband vibroacoustic reactions induced in rockets during take-off. Since then its use has become widespread and it is currently used in a multitude of different applications including shipboard noise. This method can be used to build partial or full models of noise prediction for a ship and is considered a very simple and efficient design tool. [51]. Numeric models enable us to generate sound maps for specific sections or entire decks from the given noise data for ventilation grilles, exhaust gas stacks or machinery set up in exterior areas. The great advantage of these sound maps is that critical areas can be identified, and we can monitor the possible success of any changes made. Three-dimensional modelling of deck sections and taking into account the reflection and absorption characteristics of the elements used allows mapping of acoustic conditions. The acoustic effects of screening, reflection, overlap and scattering on deck will be taken into account in the calculations so that noise measures and protection methods can be optimised during the planning phase before construction even begins. With the aid of the analyses, structural improvements can be made promptly and cost-effectively according to criteria such as transmission paths, natural frequencies, radiated sound power, dimensions or strength. Structure modifications can therefore be evaluated quickly in order to identify the optimum design.

\subsection{Statistics Energy Analysis (SEA)}

The usefulness of a particular prognostic method can be checked from evaluation of errors that result from the comparison of the predicted values with those measured on a newly built ship. Acoustic effects in small interiors are not linear and the use of simple summation of energy effects (superposition principle) is too much of a simplification. Practice confirms that discrepancies can be very large, about $+/-3$ to $5 \mathrm{~dB}$. In some cases, the differences can even reach more than ten decibels. However, even such estimates of expected vibration-acoustic risks offer great technical and economic benefits; besides, for any modern ship designed, prediction of vibration and noise levels is mandatory.

Several methods of forecasting noise on ships are currently used. A simplified empirical method given by Jansen and Buiten [52, 53] is based on the assumption that the noise level in a ship's room is determined mainly by material sounds induced during operation of ship's machinery. These sounds, when propagated within the ship's structure, shall be attenuated in proportion to internal material losses and distance from the source. Other forecasting methods described by Kihlman and Plunt [54] and Nilsson [55], Brubakk [56] and Jensen [57] were based on a similar assumption.

All these methods use the theory of wave conduction in elastic mediums and for ship issues they aim at calculating for individual decks the expected level of vibration velocity $\mathrm{L}_{\mathrm{v}}$ which will be caused by the operation of ship's main propulsion machinery. Knowing the deck vibration velocity level we can determine the vibration velocity that will occur on particular bulkheads of a compartment built in this area and then to determine the total level of noise radiated into the compartment.

One method that meets these assumptions is statistics energy analysis (SEA). This calculation method is based on a statistically simplified model of vibration distribution in complex mechanical structures. Usinga specific mathematical model, we can determine the energy transferred from the source to a further location of the structure.

Initiated by the work of Lyon and Maidanik [58], the SEA method was first used for calculations in rocket structures and later in many technical fields. Subsequent publications from the 1980s and 1990s allow to trace back the historical development of the SEA method [59, 60]. SEA is based on frequency response functions computed using a FE model of the studied structure. A large, energetic transfer matrix is computed, providing a wide observation of the vibratory behaviour of the studied structure. The knowledge of the internal loss factors defined by the user as well as numerous observation and excitation points lead to a consistent data set that may be used to properly identify a SEA model. The calculation method adopts a statistically simplified model of vibration distribution in complex mechanical structures. A specific mathematical model allows us to determine the energy transferred from the source to a further location of the structure. SEA has found common use in engineering work.

Modern development of noise prediction techniques has ensured the use of FEM and BEM methods in numerical calculations [61]. However, the optimal solution is to use hybrid FE/SEA/BEM methods in connection with SEA 
(AutoSEA2, VirtualSEA and others). For example, the hybrid model AutoSEA2 [62] contains many new advances which assist in the creation, management and solution of complex SEA models. Formulating a 3D subsystem makes the SEA modelling process more intuitive and minimizes cumulative errors to reasonable levels. The auto-connect function greatly reduces modelling time and avoids manual data input errors. Implementation of the full waveform transmission theory improves accuracy and minimizes the need for user expertise in modelling losses in nodes and discontinuities of hull structures.

Despite its unquestionable advantages, the SEA method is not devoid of some disadvantages, which is emphasized in the literature $[63,64]$. One of the most serious drawbacks of the SEA method is the omission of phase compounds occurring in the wave motion of energy transfer between systems. It is also considered as a disadvantage that this method deals with the propagation of bending waves and does not take into account the other types of waves involved and the fact that there is a limitation in its use in the low frequency range (below 200 $\mathrm{Hz}$ ), for which the condition of having a sufficient number of resonant mods is not always met.

Another problem in calculations by the SEA method is the correct choice of values of internal material loss coefficients and damping coefficients occurring in construction nodes [65]. On the way between the machine and the receiver, losses are present due to damping at each junction, starting from the first intersection, the structural sheets, until all structural discontinuities of each deck are considered. These losses should be determined in the octave band frequency range and shall depend on the loss factor of each node and the length of the structural components of the ship's hull. There are an infinite number of possible pathways between the source and the interval, which pose a problem in choosing the optimal path, the one with the lowest impedance. Therefore, lower transmission loss was considered as the shortest path between source and receiver.

Finally, there were some concerns about the acoustic radiation of vibrating structures, which are not taken into account in the SEA model. It was assumed that the problems related to radiation will result in the determination of radiation efficiency.

Despite the weaknesses of the SEA method described above, it is the most popular tool for prediction of noise on ships. Considering energy distribution by the SEA method in vibrating mechanical systems may seem quite primitive in the light of the modern expertise and applications of numerical technology. However, the main advantage of this method should be appreciated: its extraordinary simplicity of calculations conducted at an early stage of design. In the case of complex systems, it can be divided into a series of simpler subsystems with several elements, which greatly simplifies calculations.

\subsection{Numerical Packages in Shipbuilding Applications}

For the application of noise prediction on ships, extensive numerical tools are used outside the SEA method. Of many programs with acoustic modules offered on the market two programs should be distinguished: ANSYS and ACTRAN/FFT (FFT Free Field Technologies company).

ANSYS has good capabilities for solving acoustic problems and is useful in research and industrial modelling of acoustic devices. The theory behind an acoustic problem is presented, then implemented in the MATLAB code [66], solving simple systems such as acoustic field structure in free fields and progressively more complex problems such as acoustic impedance and absorptions or fluid-structure interactions (FSI). The use of finite element analysis (FEA) to solve acoustic problems has enabled investigation of complex situations that would otherwise be too hard or time consuming to solve using analytical methods. It will have a wide appeal in both academic and industrial circles. However, for many engineers, acoustics may be a new field; conversely, acousticians may not be familiar with how certain concepts are implemented in the finite element software program.

The other author's recommendation, ACTRAN, FFT's software product seems to be the most complete acoustic, vibro-acoustic and aero-acoustic software suite. ACTRAN makes up acoustic, vibroacoustic and aeroacoustic software package - Acoustics, Vibroacoustics and AeroAcoustics modules. The modelling of sound propagation includes vibroacoustic models both in the modelling space and in the radiation of physical space, characterizes the new edition of ACTRAN 2020 [67]. ACTRAN 2020 is designed to make model set-up and definition more efficient with a reorganized analysis tree, components and boundary conditions categories and a new layout and native unit conversion system offering further productivity enhancements. The model calculations shall take account of the standard acoustic test procedures for power and noise radiation measurements recommended by the IMO and ISO. The new release provides a set of tools and methods empowering users to better model advanced acoustic problems in the design of quieter products.

For one numerical model, including modal analysis of finite and infinite element method, we get the tool needed to solve the most difficult acoustic problems. ACTRAN is able to compute the equivalent aeroacoustic sources thanks to the Lighthill's analogy and Möhring's analogy and to compute their propagation taking into account the influence of the complex mean flow [68].

The strength of ACTRAN is also extensive postprocessing and graphical presentation of calculations of typical acoustic wave parameters: distributions of sound pressure, sound intensity and power generated by acoustic sources. However, very good results of FEM and BEM modelling are only achieved for airborne sounds in areas very far from the source (acoustic far field). In an acoustic near field, close to the source, modelling results are uncertain and this can be slightly improved by using hybrid models (e.g. FEM/BEM plus SEA and others). As the literature review shows, in numerical modelling of acoustic events one should think that relatively good results are obtained by the 
FWH modelling proposed by Ffowcs-Williams and Hawkings idea applied to FEM [69]. The method is based on acoustic Lighthill's analogy or the Möhring's analogy, computes the equivalent aeroacoustic sources and describes the propagation of noise in the immediate vicinity.

ACTRAN Acoustic Simulation Software, providing solutions for all industries, including shipbuilding, is developed by the Free Field Technologies company. ACTRAN/FFT offers a special Shipbuilding Applications package for the shipbuilding industry. It includes all the features required by most of the marine applications, and especially for noise predictions on new designed ships. The structural dynamic analysis of ship hulls can be performed in an external FEA solver and effectively used in prognosis calculations. The software embeds accurate acoustic simulation into design processes so ship designers can reduce noise pollution and improve crew and passenger comfort.

The Ship Acoustics Package formula is based on FEM dedicated modules and combined with several CFD codes used in the design calculations of hull dynamics parameters, based on finite and infinite elements. For extended calculation models it is possible to import vibration data from NASTRAN, ANSYS or ABAQUS to predict shipboard noise. It is possible to predict low- and high -frequency diesel engine noise, propeller and HVAC installation noise. Prognosis can be focused on providing high comfort for crew and passengers, taking particular account of interior-specific parameters affecting interior noise levels, such as room-toroom insulation, sound radiation efficiency, the acoustic radiation from vibration floors, walls and ceilings. The results shall be given as the total effect of the ship's structureand airborne sound using a logarithmic measure of the levels given in $\mathrm{dB}(\mathrm{A})$. The calculation of the predicted parameters should be in accordance with internal standards set by the government agencies specializing in ship and platform construction. The fulfilment of the standards will depend on the classification society that supported their construction and the regulations of the country where the ship is to be registered.

\section{Summary and Conclusions}

The main objective of the article was to describe new, highly effective research methods for protection against excessive noise in enclosed spaces rooms on merchant and passenger ships. The non-conventional testing techniques set out in the article concern the study of acoustic hazards occurring in ships' spaces, subject to IMO and ISO regulations. For new vessels of the Quiet Ship class, compliance with proper acoustic protection becomes a major problem for designers of new ships friendly to people and the environment.

The innovation of the research proposed in the paper remains in two general areas:

1) Scientific development - theoretical work carried out within the framework of the new sound theory (VST), which is currently being developed, and the application of this theory in the research on vector sound field distributions using the sound intensity technique (SIAOD) and laser image anemometry (A-PIV, A-LDA),

2) Industrial applications - vibration-acoustic optimisation of new products that generate excessive noise in the environment at levels exceeding standard limits.

Introduction of the new Vortex Sound Theory to the modern acoustics allows us to complete the current level of knowledge about the genesis of sound and the distribution of acoustic fields. Wave descriptions of acoustic events can have a vector form while theoretical and experimental studies of the energy characteristics of acoustic flows can be conducted like those in fluid mechanics.

In acoustic flows, the visualization of the structural distribution of acoustic fields, shown in a vector form on in plane or spatial images $(2 \mathrm{D}, 3 \mathrm{D})$, is an innovative technique that permits us to explain the mechanism of sound generation in disturbed flows and reveals the structural nature of acoustic flow (acoustic coherent structures like layered liquid streams). Sound visualization in acoustic wave flows reveals the predominant whirling nature of acoustic flow. These are new elements of the theory of acoustic fields, different from those used in classical acoustics.

New methods consisting in the recording of vector parameters of wave motion enable illustrating the results of vibroacoustic research by the Acoustic Imaging technique and presenting the results of measurements as maps of acoustic energy distributions in real fields. Viewing the noise images will make it easier for designers and constructors to choose effective technical methods to reduce the risk of noise on board a ship.

It should be emphasized that there are significant differences between the research techniques used in classical acoustics and the methods proposed in the article. The new SI, AOD, A-PIV/A-LDA testing techniques presented herein, together with the visualization of the results by the AI method, are based on the research of vector parameters of the acoustic wave and describe the energy effects of wave flows.

The energetic characteristics of ship noise should also be taken into account when selecting noise prediction methods for newly designed ships. Although the modified SEA method (AutoSEA2) has become common in prediction of noise in shipboard rooms, according to the author, much higher prediction accuracy is obtained by using FEM/SEA/BEM hybrid methods, in which the use of SEA model statistical data is only a very limited auxiliary part of calculations.

The final conclusions of the paper can be summarised in several points. General possibilities of new techniques in marine noise research are as follows:

1) Conducting energy analyses of the effects that occur in real acoustic fields and the possibility of direct measurement of the acoustic power of industrial sound and noise sources;

2) Analysing the disturbed acoustic flows in a vector form, that is hydrodynamic and aeroacoustic phenomena using the knowledge available from fluid mechanics; 
3) Illustrating the results of vibroacoustic research; Acoustical Imaging technique can be used to present maps of acoustic energy distributions $(2 \mathrm{D}, 3 \mathrm{D})$ in real fields;

4) Showing the trajectory of wave motion, the real effects of reflection, refraction and scattering of the wave on obstacles introduced into the acoustic field, to show the mechanism of sound transmission through partitions, and to study acoustic phenomena in real-life environments never seen before as the results of experimental research;

5) Significant improvement of the technique of diagnosing equipment with acoustic methods and precisely indicate all local noise sources on the tested structure or determine the causes of noise occurring in confined spaces, indicating areas with the lowest acoustic impedance on the partitions and places of lateral penetration (hot spots and acoustic holes);

6) Experimental check, validation and verification of the reliability of the results of the vortex and turbulent flow tests obtained from numerical modelling carried out by FEM, BEM, SEA methods used on CFD/FSI/CAA platforms.

In a separate publication, the author will present extended results of shipboard tests using the SI-AOD method discussed in this article. It will show the research of vibroacoustic parameters of the ship's cabin prototypes installed in the ship's superstructure mock-up model, which will allow the designers to improve the prototype in terms of noise protection and to start mass production of components meeting the standard and requirements of classification societies. Detailed tests of cabin sections, such as walls with windows and doors, floors and ceilings, provide full information on the vibro-insulating characteristics of these components. Much attention will be paid to the installation of marine HVAC systems, the third major source of ship's noise. Finally, we will demonstrate the use of SI-AOD technology on a vessel ready to be delivered to the shipowner for operation.

\section{Declaration of Competing Interest}

The author declare that they have no known competing financial interests or personal relationships that could have appeared to influence the work reported in this paper.

\section{References}

[1] EU Ecolabel Procedures, www.ecolabel.eu, 2019 (accessed 5 Nowember 2019).

[2] IMO - Code on noise levels on Board Ships - Res. A.468 (XII), SOLAS 2014.

[3] EU Marine Directive. The European Marine Strategy Framework Directive (MSFD) requires uropean states to maintain their marine waters in 'Good Environmental Status' (GES). European Commission, 2010.
[4] DNV GL. Rules for classification. Ships. Part 6 Additional class notations. Chapter 7 Environmental protection and pollution control, 2015.

[5] Lloyd's, Register - An executive agency of the Department for Transport - Code of Safe Working Practices for Merchant Seamen Consolidated Edition. London 2010.

[6] Boure Veritas - NR614 Underwater Radiated Noise (URN), 2014.

[7] RINA (Registro Italiano Navale). Rules for the Classification of Ships: Amendments to Part F: Additional Class Notations, 2014.

[8] Erbe C. at al. The Effects of Ship Noise on Marine Mammals - A Review. Frontiers in Marine Science, 2019.

[9] Lighthill M. J., On sound generated aerodynamically 1: General theory, Proc. Of the Royal Society of London, A 211, 1952 p. 564-587.

[10] Curle N., The influence of solid boundaries upon aerodynamic sound., Proc. Of the Royal Society of London, A 23, 1955 p. 505-514.

[11] Powell A., Theory of vortex sound. J. of the Acoustical Society of America 36: 1964 p. 177-95.

[12] Goldstein M. E., Aeroacoustics, McGraw-Hill, 1976.

[13] Ffows-Williams J. E., Aeroacoustic. Journal of Sound and Vibration 190 (3), 1969 p. 387-398.

[14] Möhring W., On vortex sound at low Mach number. Journal of Fluid Mechanics 85, 1978, p. 685-691.

[15] Howe M. S., Theory of Vortex Sound. Cambridge: Cambridge University Press, 2003.

[16] Kambe T. Vortex sound with special reference to vortex rings: theory, computer simulations, and experiments. Aeroacoustics $9(1,2), 2010$ p. $51-89$.

[17] Weyna S., Some comments about the existing theory of sound with comparison to the experimental research of vector effects in real-life acoustic near fields. Archives of Acoustics Vol. 32 (4), 2010 p. 441-451.

[18] Hansen C., Foundations of Vibroacoustic. CRC Press 2018.

[19] Tang S. K., Ko NWM. Sound generation by interaction of two inviscid two-dimensional vortices. The Journal of the Acoustical Society of America Vol. 102. 1997 p. 14631473.

[20] Schram C., Hirschberg A. Application of vortex sound theory to vortex-pairing noise: sensitivity to errors in flow data. Journal of Sound and Vibration, 266, 2003 p. 1079-1098.

[21] Tropea A., Yarin AL, Foss JF. Springer Handbook of Experimental Fluid Mechanics, Springer-Verlag, Berlin, Heidelberg 2007.

[22] Hafez M.. at al. Computational Fluid Dynamics Review 1995. Wiley \& Sons, Chichester, 1995.

[23] Fahy, F. J., Sound Intensity. Elsevier Applied Science, London 1989.

[24] de Bree H-E, at al, Three-dimensional sound intensity measurement using microflown particle velocity sensors. MEMS'99, Orlando 1999. 
[25] Dickinson R. R., A unified approach to the design of visualization software or the analysis of field problems, Proc. Three-dimensional Visualization and Display Technologies, 1989 p. $173-180$.

[26] Schroeder W., at al, The Stream Polygon: a Technique for 3D Vector Field Visualization. Proc. of Visualization '91, San Diego, 1991 p. 26-132.

[27] Weyna S. Acoustics flow field visualization using sound intensity and, laser anemometry methods, Proceedings of XX Fluid Mechanics Conf.; KKMP2012, S27-2, 2012.

[28] Gerald-Yamasaki M., Visualization of Computational Fluid Dynamics. Comp. Fluid Dynamics - Rev. 1995.

[29] Nowicki A. Ed., Acoustic Imaging. Springer, Vol. 312012 , (Weyna S. - chapter; Visualization method of acoustic wave propagation based on the sound intensity measurement, p. 243-252.

[30] Schuster H. G., Deterministic Chaos. An Introduction. VCH Verlagsgesellshalf 1988.

[31] Ruelle D. Ed., Turbulence, Strange Attractors and Chaos. Nonlinear Science, France 1995.

[32] Williams E. G., Fourier Acoustics - Sound radiation and nearfield holography. Academic Press. San Diego, London, New York 1999.

[33] Weyna S., Acoustic intensity imaging methods for in-situ wave propagation, Archives of Acoustics, 35, 2, 2010 p. 265273.

[34] Hain R., Kähler Ch. J., Michaelis D. - Tomographic and time resolved PIV measurements on a finite cylinder mounted on a flat plate. Exp. Fluids, Vol. 45: 2008 p. 715-724.

[35] Haigermoser Ch., Application of an acoustic analogy to PIV data from rectangular cavity flows. Exp. Fluids Vol. 47, 2009 p. $145-157$.

[36] Adrian, R. J, Yao C. S., Power spectra of fluid velocities measured by laser Doppler velocimetry. Exp. in Fluids, Vol. 5: 1987 p. $17-28$.

[37] Nobach H., Müller E., Tropea C., Efficient estimation of power spectral density from laser Doppler anemometer data. Experiments in Fluid Vol. 24 (5/6), 1998 p. 499-509.

[38] Valière J-Ch., Moreau S., Bailliet H., Development of Laser Techniques for Acoustic Boundary Layer Measurements. Part I: LDV Signal Processing for High Acoustic Displacements. ACTA ACUSTICA united with ACUSTICA, Vol. 95, 2009 p. 585-594.

[39] Raffel M., Willert C., Kompenhans J., Particle image velocimetry. A practical guide. Springer, Heidelberg 1998.

[40] Zhang Zh., LDA Application Methods - Laser Doppler Anemometry for Fluid Dynamics. Springer, 2010.

[41] Moreau S., Bailliet H., Valière J-Ch., Boucheron R. Development of Laser Techniques for Acoustic Boundary Layer Measurements. Part II: Comparison of LDV and PIV Measurements to Analytical Calculation. ACTA ACUSTICA united with ACUSTICA, Vol. 95, 2009 p. 805-813.

[42] Hain R., Kahler Ch., Fundamentals of multiframe particle image velocimetry (PIV). Exp. Fluids Vol. 42: 2007 p. 575-587.
[43] Macdonald R., Skulina D., Campbell M., Valerie J-Ch., Marx D., Bailliet H. PIV and applied to high amplitude acoustic field at a tube termination. Congres Francais d'Acoustique, 2010 p. $10-16$.

[44] Weyna S, Mickiewicz W, Pyla M, Jablonski M. Experimental Acoustic flow analysis inside a section of an acoustic waveguide, Archives of Acoustics Vol. 38 (2), 2013 p. 211216.

[45] Fischer R., Boroditsky L., Design guide for shipboard airborne noise control. SNAME Technical \& Research Bulletin 3-37, Noise Control Engineering, 2001.

[46] Schmid P. J., Dynamic mode decomposition of numerical and experimental data Journal of Fluid.

[47] Ffowcs-Williams, J. E., Hawkings DL. Sound Generation by Turbulence and Surfaces in Arbitrary Motion. Philosophical Transactions of the Royal Society, 2641969.

[48] Lu H. Z., Lixi H., So R. M. C., Wang J., A computational study of the interaction noise from a small axial-flow fan. The Journal of the Acoustical Society of America Vol. 110 (5), 2007,4041415 .

[49] Weyna S., Visualizations of the subsonic acoustic wave flow inducing noise inside open ducts. Proc. $20^{\text {th }}$ International Congress on Sound and Vibration, Bangkok 2013.

[50] Lyon, R. H., Statistical Energy Analysis of Dynamical Systems, MIT Press, 1975.

[51] Plunt J., Methods for Predicting Noise levels in Ships. Part II: Prediction of Structure-borne Sound Transmission in Complex Structures with the SEA Method. PhD Thesis, Dept. Building Acoustics, Chalmers University, 1975.

[52] Jensen J., Calculation of structure-borne sound in plate structures by the application of "Statistical Energy Analysis”Theoretical and experimental study. Proc. NAS-78 Meeting, 1978, p. 165-1678.

[53] Buiten J., Aarsten H. Simplified method for predicting sound in a accommodation spaces aboard sea going motorships. Proc. Inter-Noise'79, 1979.

[54] Kihlman T., Plunt J., Noise prediction in ships. ASA Meeting, Austin 1975.

[55] Nilsson A. C., Noise Prediction and Prevention in Ships. Sem. über Festigkeit Schwingungen Lärm, Hamburg 1977.

[56] Brubakk E., Acoustical planning in ship design. DNV. Etan Marine Sympozjum 80’, Zadar 1980.

[57] Jensen J., - Noise in the accommodation on board ships. Proc. „,The Danish Days of Science”, Leningrad 1975.

[58] Lyon R. H., Maidanik G., Power flow between lineary coupled oscilators. J. Acous. Soc. Am., 34, 1962 p. 623-631.

[59] Bremner P., Nelisse H., Shorter P., An Overview of AutoSEA 2. Canadian Acoustics Vol. 28 (3) (2000), 166-174.

[60] Egab L., Wang X., Fard M., Jazar R., Prediction of interior trim sound absorption using Statistical Energy Analysis. Proceedings of ISMA2012, 3871-3877.

[61] Mace B. R., Shorter P. J., Energy Flow Models from Finite Element Analysis, Journal of Sound and Vibration 233 (3), 2000 p. 369-389. 
[62] ESI Group - SEA/AutoSEA2 module, User's Guide, 2007.

[63] Rosen M,. Borello G., Damping and coupling loss factors estimation in SEA method: what is really measured? InterNoise 1996.

[64] Weyna S., Universal methodology for noise predicting on newly designed ships. Part II, Szczecin University of Technology, 1984 (in Polish).

[65] Gagliardini L., Houillon L., Virtual SEA - FEA-based modelling of mid-frequency structure-borne noise. Sound and Vibration 2005 p. 22-28.
[66] Howard C. Q., Cazzoloto BS. Acoustic Analyses Using MATLAB and ANSYS. CRC Press 2017.

[67] ACTRAN: The Gold Standard in Acoustic - Ship acoustic analysis - www.fft.be, 2019 (accessed 10 December 2019).

[68] Zhang Y., Zhang T., Ouyang H., Li T., Flow-induced noise analysis for 3D trash rack based on LES/Lighthill hybrid method. Applied Acoustics, Vol. 79, 2014, p. 141-152.

[69] Escobar M., Ali I., Hahn C., Kaltenbacher M., Numerical and experimental investigation on flow induced noise from a square cylinder. 10th AIAA/CEA, 2012 AIAA2004-3004. 